\title{
Erla Hallsteinsdóttir*
}

\section{Phraseographie}

\begin{abstract}
Multiword expressions - i.e. phraseological units - like idioms and collocations are one of the most interesting part of every language. In this article, I investigate phraseological units from a lexicographical point of view. I discuss the theoretical and methodological basis of phraseography as a discipline that includes aspects of lexicography, phraseology, corpus linguistics and theories of language learning. I demonstrate the importance of corpora as a source for the lexicographer and the use of corpus data. I also discuss the requirements for the lexicographical treatment of phraseological units by the compilation of a phraseological database for language learners in relation to their assumed needs that have already been described in detail.
\end{abstract}

\section{Einführung}

Unabhängig davon, ob man Phraseologismen als gewöhnliche sprachliche Einheiten, die "nicht komplizierter und weniger notwendig als andere sprachliche Einheiten" (Kühn 1992: 172) sind, oder als "das Sahnehäubchen des elaborierten Umgehens mit einer Sprache" (Harras 1997: 52) betrachtet, sind ein- und mehrsprachige Nachschlagewerke ein unerlässliches Hilfsmittel beim Lernen, beim Verstehen sowie beim Verwenden und Übersetzen von Phraseologismen. Dies gilt nicht nur für Fremdsprachenlerner, sondern auch für die Beherrschung der Phraseologie in der Muttersprache sind adäquate lexikographische Nachschlagewerke wichtige Hilfsmittel.

Das Forschungsgebiet, das sich mit theoretischen Aspekten der Phraseologie im Hinblick auf die Darstellung von Phraseologismen in Wörterbüchern und mit Phraseologismen in der praktischen Wörterbucharbeit befasst, nennt man Phraseographie. Die Phraseographie als Forschungsgebiet und als praktische Wörterbucharbeit vereint Frage-

\footnotetext{
* Erla Hallsteinsdóttir Veegtense Kvarter 336

DK-5220 Odense SØ

erlahallsteinsdottir@web.dk

Hermes - Journal of Language and Communication Studies no 36-2006
} 
stellungen und Forschungsthemen aus mehreren Disziplinen. In diesem Artikel sollen ausgewählte Bereiche der Phraseographie diskutiert werden, die an Schnittstellen zu folgenden Forschungsgebieten thematisiert werden:

1. Die erste Schnittstelle führt zur Grundlagenforschung in der Phraseologie, deren Ergebnisse, sowohl in Form von theoretischen Arbeiten als auch Methodik und konkreten Beschreibungen, die Grundlagen für phraseographische Arbeiten liefert.

2. Die zweite Schnittstelle berührt die Lexikographie mit den theoretischen Grundlagen der Wörterbuchforschung, der Wörterbuchbenutzungsforschung und der Methodik der praktischen Wörterbucharbeit.

3. Die dritte Schnittstelle bezieht sich auf phraseographische Werkzeuge, die der Phraseographie mit Theorien und Methoden der Korpuslinguistik (und Sprachtechnologie allgemein) zur Verfügung stehen.

4. Die vierte hier behandelte Schnittstelle ist die Phraseodidaktik. Hier geht es um Benutzungssituationen für phraseologische Wörterbücher und die Bedürfnisse potentieller Benutzergruppen.

Die übergeordnete Perspektive der folgenden Darstellungen ist eine anwendungsorientierte im Bezug auf Fremd- und Muttersprachenlernen und Übersetzen. Diese wird geprägt durch meine (meta)sprachlichen Erfahrungen im täglichen familiären und fachlichen Umgang mit den drei Sprachen Isländisch (als Muttersprache), Deutsch und Dänisch (als Fremdsprachen), die hier auch als Beispielsprachen herangezogen werden. Die Beispiele stammen, wenn keine andere Quelle angegeben wird, aus den Korpora von Deutscher Wortschatz (www.wortschatz.unileipzig.de)

\section{Grundlagen der Phraseographie}

\subsection{Phraseologie}

Den Gegenstandsbereich der Phraseographie bildet die Phraseologie, definiert als das Inventar einer Sprache an Phraseologismen. Die phraseographische Arbeit an und mit Phraseologismen beruht auch zum größten Teil auf den Ergebnissen der Phraseologie als Forschungsgebiet (vgl. Burger 1998: 11 zur Doppeldeutigkeit des Terminus Phraseologie). Für 
den Gegenstandsbereich der Phraseographie sollte vom "Bereich der Phraseologie im weiteren Sinne" (Burger 1998: 14) ausgegangen werden, denn nicht nur der Teilbereich der Idiome gehört zum Aufgabenbereich der Phraseographie, sondern alle Phraseologismen (und in einigen Fällen sogar auch Parömien). Demnach wird hier folgende Definition verwendet:

Ein Phraseologismus ist eine (relativ) feste Mehrwortverbindung unterhalb der Satzebene, deren Einzelkomponenten gemeinsam eine (möglicherweise von der additiven isolierten Bedeutung der Komponenten abweichende) lexikalisierte Bedeutung haben. Dieser Definition liegen folgende Voraussetzungen zugrunde:

(a) Das grundlegende Merkmal der Definition ist die äußere Form als eine feste Verbindung von mehr als einem Wort.

(b) Die Phraseologie umfasst nur Phraseologismen unterhalb der Satzebene. Satzwertige Mehrwortverbindungen sind Gegenstand der Parömiologie. Pilz (1987: 129) geht zwar von "einer "Phraseologie im wei(tes)ten Sinne" aus", die auch Parömien umfasst, die Zweiteilung nach dem Kriterium der Satzwertigkeit hat sich jedoch in der Forschung durchgesetzt.

(c) Die Idiomatizität ist ein semantisches Merkmal, das nur in einem Teilbereich der Phraseologie relevant ist, und deshalb nicht übergeordnet für die Abgrenzung der Phraseologismen von anderen sprachlichen Einheiten verwendet werden sollte. Es ist außerdem deshalb problematisch, Phraseologismen von der Semantik ausgehend zu definieren, weil mit den gegenwärtigen Forschungsergebnissen weder die isolierte Bedeutung der Komponenten, die phraseologische Bedeutung noch pragmatische Aspekte der Bedeutungen eindeutig definierbar sind.

(d) Es gibt keine Einwortphraseologismen, wohl aber idiomatische Wörter, die in einer engen Relation zu Phraseologismen stehen können, wie Dünnbrettbohrer und das Brett bohren, wo es am dünnsten ist. Ein Ausschluss der idiomatischen Wörter (als Einwortlexeme) aus dem Bereich der Phraseologie per definitionem bedeutet nicht, dass diese in der Phraseologie keine Rolle spielen und phraseographisch nicht berücksichtigt werden sollten. Wenn Relationen zwischen Phraseologismen und Einwortlexemen bestehen, müssen sie, als Teil der Beschreibung der betreffenden Phraseologismen, er- 
fasst werden. Diese Beziehungen sind aber auch exemplarisch für eine generelle Problematik der isolierten Betrachtung von Phraseologismen, in der die vielfältigen Relationen von Phraseologismen $\mathrm{zu}$ anderen sprachlichen Einheiten kaum berücksichtigt werden.

Die Phraseologieforschung hat inzwischen einiges an theoretischer Vorarbeit für die Phraseographie geleistet. Es gibt eine Reihe von theoretischen Arbeiten wie z. B. zum Wesen der Phraseologie im Sprachsystem und in der Sprachverwendung, unterschiedliche Klassifikationsmodelle, semantische und grammatisch-syntaktische Beschreibungsansätze sowie viele Einzelstudien zu und Analysen von bestimmten Bereichen in der Phraseologie, die die konkrete inhaltliche Grundlage für phraseographische Arbeiten bilden. Hier gibt es aber eine Reihe ungelöster Probleme, die für die Phraseographie relevant sind:

Es gibt noch keine einheitliche Terminologie für die Phraseologie. Es existieren fast so viele terminologische Ansätze wie es theoretische Auffassungen gibt. Die heterogene theoretische Basis führt zu heterogenen Klassifikationsansätzen innerhalb der Phraseologie, die die Einordnung von Phraseologismen zu entsprechenden Beschreibungsmodellen erschwert. Die teilweise sehr unterschiedlichen theoretischen Auffassungen über wesentliche Eigenschaften von Phraseologismen wie z. B. semantische Eigenschaften, die Beschaffenheit einer phraseologischen Kulturspezifik, phraseologische Bilder, die Beschreibung pragmatischer und morpho-syntaktische Aspekte, die (mentale/systembezogene) Festigkeit, die mentale Repräsentation und Verarbeitung usw. sind große Hürden für die Arbeit an adäquaten Modellen für die phraseographische Erfassung und Beschreibung der Phraseologie.

Intrasprachliche systematische Erfassungen und Beschreibungen von Phraseologismen und phraseologischen Relationen im Wortschatz sind noch sehr lückenhaft.

Die wichtigste Aufgabe der Phraseologieforschung besteht in einer präzisen systematischen Beschreibung der Phraseme aller Klassen in allen möglichen Sprachen. Paradoxerweise hat sich die Phraseologieforschung mit dieser Aufgabe noch nie ernsthaft befaßt, wahrscheinlich in der Annahme, daß die Wörterbuchmacher sie schon irgendwie bewältigen würden. Die Vernachlässigung der "niedrigen Empirie" zugunsten der "hohen Theorie" hat dazu geführt, daß es trotz der jahrzehntelangen aktiven Tätigkeit vieler Phraseologieforscher in verschiedenen Ländern der Welt kein einziges (mir bekanntes) Wörter- 
buch gibt, in dem der Benutzer die notwendigen Informationen für die produktive (und nicht nur rezeptive) Beherrschung der Phraseme finden kann. (Dobrovol'skij 2002: 429)

Noch größere Lücken bestehen bei intersprachlichen Vergleichen von Phraseologismen, die für die zwei- und mehrsprachige Phraseographie unentbehrlich sind.

Ein Blick auf die hier verwendeten Beispielsprachen zeigt jedoch, dass schon ernste - teils phraseographisch motivierte - Anstrengungen gemacht werden, um diese Lücken zu füllen. Für die deutsche Phraseologie wird in dem von der Alexander von Humboldt-Stiftung mit dem Wolfgang Paul-Preis geförderten Forschungsprojekt "Kollokationen im Wörterbuch" an einer umfassenden computergestützten Beschreibung von Phraseologismen gearbeitet (vgl. Fellbaum et al. in Vorb. und www.bbaw.de/forschung/kollokationen.html). Im Entstehen ist eine phraseologische Datenbank, die auch als Grundlage für phraseographische Arbeiten zur Verfügung stehen wird. Weiterhin gibt es einige elektronische Projekte, die sich mit einzelnen Arten von Phraseologismen befassen, wie die "Sammlung unikaler Wörter des Deutschen" an der Universität Tübingen (www.sfb441.unituebingen.de/a5/codii/index.xhtml) und eine Untersuchung deutscher Phraseologismen ohne freie Lesart an der Universität Jena (www.personal.uni-jena.de/ xcr/IVP-Web/ivp-liste-X.xml) - um einige Projekte zu nennen, zu denen aktuelle Forschungsberichte vorliegen.

In der isländischen Phraseologie liegen neuere gedruckte phraseologische Wörterbücher von Jónsson (2001 und 2002) vor, deren umfassende lexikographische Grundlage als eine z. Z. unveröffentlichte Datenbank existiert. Die dänische Phraseologie hat in den letzten Jahren einen Aufschwung erlebt (vgl. Farø 2005), u. a. mit einem speziell für das Internet konzipierten Idiomwörterbuch (www.idiomordbogen.dk; vgl. auch die Rezension in Farø 2004a). Die korpusgestützten Arbeiten von Farø (vgl. z. B. 2004b) bilden eine Basis für die dänisch-deutsche Phraseographie. Für die dänisch-isländische Phraseologie steckt ein Forschungsprojekt unter der Leitung von Auður Hauksdóttir an der Universität in Island in den Anfängen. Im Bereich der deutsch-isländischen Phraseologie arbeite ich an einem Forschungsprojekt, als dessen Ergebnis eine zweisprachige Phraseologiedatenbank entstehen soll (vgl. Hallsteinsdóttir im Druck; Hallsteinsdóttir in Vorb. a+b). 


\subsection{Lexikographie}

Die Lexikographie ist die zweite Disziplin, auf der die Phraseographie aufbaut. Die Lexikographie wird als ein selbständiges Forschungsgebiet der Wörterbuchforschung angesehen, das neben lexikographischen Theorien über den Aufbau und den Inhalt von Wörterbüchern und der Methodik der praktischen Wörterbucharbeit weitere Aufgabengebiete umfasst, wie z. B. die Erstellung von Wörterbuchtypologien, die historische Wörterbuchforschung und die Wörterbuchbenutzungsforschung.

Eine wichtige Forschungsrichtung der Lexikographie befasst sich mit Wörterbüchern und Wörterbuchinhalten im Bezug auf die Bedürfnisse von Wörterbuchbenutzern in bestimmten Benutzungssituationen. Wörterbücher werden als Gebrauchsgegenstände aufgefasst und diese Auffassung bildet den Ausgangspunkt für sowohl lexikographische Theorien als auch die praktische Wörterbucharbeit. Die grundlegende lexikographische Frage ist dementsprechend die Frage danach, welche Benutzer welche Wörterbücher in welchen Situationen zu welchem Zweck benutzen (vgl. dazu z. B. Bergenholtz/Tarp 2003).

Die Computerlexikographie stellt inzwischen einen wichtigen Teilbereich der Lexikographie dar. In den letzten Jahren ist der lexikographische Bedarf an Wissen im Bereich der Sprachtechnologie stark gestiegen, so dass sich Lexikographen zunehmend mit informatikwissenschaftlichen Aspekten (Hypertextlexikographie, Datenbanksoftware) und Computertechnik auseinandersetzen (müssen). Dies schließt Theorien und Methoden der Korpuslinguistik mit ein, auf deren Beitrag zur Phraseographie im Abschnitt 2.3 eingegangen wird.

Eine Entwicklungstendenz in der Computerlexikographie geht in die Richtung, dass dynamische Informationssysteme aufgebaut werden sollen, die mit Hilfe empirischer Daten (gewonnen z. B. mit Kookkurenzanalysen oder anderen korpusbasierten Verfahren) möglichst viele Aspekte einer Sprache umfassen und genau beschreiben sollen (siehe z. B. die Projekte ELEXIKO (www.elexiko.de) und LEKSIS am IDS in Mannheim (www.ids-mannheim.de)) (vgl. auch Fraas/Haß-Zumkehr 1998). Ein Aspekt dieser Tendenz ist, dass die traditionelle Aufteilung von Wörterbüchern nach dem sprachlichen und/oder fachlichen Inhalt aufgehoben und die Inhalte mehrerer Wörterbücher vereint werden. Jeder Wörterbuchbenutzer soll dann aus der umfassenden Beschreibung der Sprache einfach die Wörterbuchinformationen heraussuchen, die in 
der jeweiligen Benutzungssituation seinen Bedürfnissen entsprechen (vgl. Bergenholtz 1997 zur Polyfunktionalität von Wörterbüchern). Die technischen Voraussetzungen dafür, dass jeder Wörterbuchbenutzer aus dem Informationsangebot eines lexikalischen Informationssystems individuelle Wörterbücher zusammenstellen könnte, wären schon vorhanden.

Erste Überlegungen zu den Möglichkeiten der Computerlexikographie in der Phraseologie sind schon bei Pilz zu finden, der für die Erarbeitung eines umfassenden Phraseolexikons mit "sinnvollem Computereinsatz" (Pilz 1987: 141) plädiert. Ebenfalls ist bei Wotjak (1992: 56) der Hinweis auf die Eignung ihrer Mehrebenenbeschreibung für "eine Computerimplementierung" zu finden, die sowohl bei der intraals auch bei der interlingualen Beschreibung von Phraseologismen neue Möglichkeiten eröffnen würde. Die erste Arbeit in der deutschen Computerphraseographie lieferte Dobrovol'skij mit seiner Datenbank deutscher Idiome (mit ca. 1000 Idiomen, vgl. Darstellung in Keil 1997: 164 ff.). Dem folgen die Arbeiten von Keil (1997) mit einer detaillierten Beschreibung der Computerimplementierung von Phraseologismen in der Datenbank Phraseo-Lex und das deutsch-französische Forschungsprojekt CONPHRAS mit einer phraseologischen Hypertext-Datenbank (vgl. Gréciano 1997). Weiterhin gibt es mit dem AUTOFRAS-Projekt (vgl. Pamies et al. 1998) die Vorarbeit für eine experimentelle Datenbank für Phraseologismen in 10 Sprachen mit einer onomasiologisch ausgerichteten Beschreibung.

Ein elektronisches Phraseologiewörterbuch, das gezielt alle Möglichkeiten der Computerlexikographie in Anspruch nimmt - Mehrdimensionalität, Hypertext und benutzerbezogene Informationshierarchien, Verwendung von audiovisuellen Medien, die Kombination von Onomasiologie und Semasiologie usw. - steht allerdings immer noch aus. Die vorhandenen theoretischen und praktischen Ansätze für die Computerphraseographie (vgl. Fellbaum et al. in Vorb., Hallsteinsdóttir in Vorb. $\mathrm{a}+\mathrm{b}$ und Piirainen 2000a und 2000b) müssten nach einem Tauglichkeitstest in der Praxis als Grundlage für die lexikographische Arbeit in einem einheitlichen Wörterbuchkonzept zusammengefasst werden.

\subsection{Korpuslinguistik}

Textkorpora sind eine wichtige Ressource für Phraseographen. Mittlerweile gibt es ein breites Angebot an wissenschaftlichen Textkorpora 
im Internet. Viele sind frei zugänglich und die meisten sind benutzerfreundlich und einfach zu bedienen. Beispiele für solche Korpora sind z. B. das Projekt Deutscher Wortschatz (www.wortschatz.unileipzig.de), Das Digitale Wörterbuch der deutschen Sprache des 20. Jahrhunderts (www.dwds.de) und die Korpora des Instituts für Deutsche Sprache www.ids-mannheim.de/kt/corpora.html für Deutsch, das Korpus 2000 für Dänisch (www.korpus2000.dk) und die Korpora des lexikographischen Instituts an der Universität in Island für Isländisch (www.lexis.hi.is/corpus/leit.pl).

Textkorpora können u. a. auf folgende Fragen in Verbindung mit der Phraseographie eine Antwort geben:

- Wie häufig kommt ein Phraseologismus in einem bestimmten Textkorpus vor?

- In welchen Kontexten kommt ein Phraseologismus vor (Textsorten, Sprecher, Themen)?

- Welche Bedeutung(en) hat ein Phraseologismus in den Korpusbelegen (Polysemie, Bedeutungsvariationen)?

- Gibt es zu einem Phraseologismus eine formgleiche nicht-phraseologische Konstruktion der Komponenten (i. e. "wörtliche" Bedeutung(en))?

- Gibt es (z. B. semantisch-pragmatische oder morpho-syntaktische) Präferenzen oder Restriktionen für die Verwendung eines Phraseologismus?

- Welche Verwendungsformen des Phraseologismus kommen vor (i. e. Variantenvielfalt)?

Im Folgenden wird der Nutzen von Korpora in der Phraseographie anhand von zwei Themen dargestellt: (a) Frequenzbestimmungen in Textkorpora als eine Grundlage für die Auswahl von Phraseologismen, die in ein Wörterbuch aufgenommen werden, und (b) Überprüfung lexikographischer Informationen durch Analysen von Korpusbelegen.

\subsubsection{Frequenzbestimmungen von Phraseologismen in Textkorpora}

Ein häufig kritisierter Aspekt bei Wörterbüchern ist die fehlende Beschreibung der Grundlage für die Auswahl der aufgenommenen Phra- 
seologismen. In vielen Wörterbüchern basiert die Auswahl wohl primär auf der subjektiven Sprachkompetenz und der Intuition der Wörterbuchautoren. Diesem Verfahren gegenüber steht die Forderung nach der Verifizierung der Auswahl durch objektive empirische Daten.

Inzwischen liegen bereits Ergebnisse zur Frequenz von Phraseologismen als Angaben zur relativen Frequenz einzelner Phraseologismen auf eine Million Wörter (PMW-Angaben) in einigen Sprachen wie Tschechisch (vgl. Čermák 2003), Französisch, Niederländisch (vgl. Colson 2003) und Englisch (vgl. Cowie 2003) vor. Diese Ergebnisse wurden in der Regel dadurch gewonnen, dass spezielle Wortkombinationen ausgewählt und auf ihr Vorkommen im Korpus überprüft wurden. Die Untersuchungen kommen trotz aller Objektivität nicht ohne die subjektive Sprachkompetenz der Wissenschaftler aus, denn es muss geeignetes Ausgangsmaterial gefunden und aufbereitet werden. Außerdem müssen die Ergebnisse einer Korpusanalyse ausgewertet und interpretiert werden, denn bei einigen Phraseologismen kann eine formgleiche Konstruktion in nicht-phraseologischer Bedeutung vorkommen und es gibt $z$. T. mehr als einen Phraseologismus zu den verwendeten Suchformen, so dass eine manuelle Analyse der Korpusbelege in der Regel notwendig ist.

Frequenzanalysen geben Auskunft darüber, wie häufig die dafür konstruierten Suchformen der Phraseologismen in Texten vorkommen. In der Forschung herrscht aber allgemein Einigkeit darüber, dass sie nicht für sich allein als Auswahlkriterium für Phraseologismen verwendet werden sollen. Als das wichtigste Kriterium für die Auswahl von Phraseologismen für zweisprachige Wörterbücher sieht Dobrovol'skij die Geläufigkeit bei Muttersprachlern. In Bezug auf die Angabe von äquivalenten Phraseologismen im Wörterbuch betont er, dass das Kriterium der Geläufigkeit auf die aufzunehmenden Idiome beider Sprachen angewendet werden muss (vgl. Dobrovol'skij 1997: 48). Die Wichtigkeit dieses Prinzips möchte ich am folgenden Beispiel zeigen. Für den deutschen Phraseologismus jmd <eine Frau> hat die Hosen an ('ist Herr im Hause') (vgl. DUDEN 11: 350) wird im deutsch-isländischen Wörterbuch das Äquivalent hún er á báðum buxunum angegeben (Ófeigsson 1982:331) (Bedeutungsangabe in Friðjónsson (1993: 90): 'jemand hält viel von sich selbst' mit der Markierung 'selten'). Es mag sein, dass dieser isländische Phraseologismus bei der Erstausgabe des Wörterbuchs im Jahre 1935 geläufig war, heute dürfte er aber den 
100

meisten Muttersprachlern unbekannt sein (Ófeigssons Wörterbuch ist das einzige "große" deutsch-isländische Wörterbuch und es wird noch immer - mehr oder weniger unverändert - verkauft). Ein funktional äquivalenter Phraseologismus wäre z. B. einhver er húsbóndi á sínu heimili.

Die Geläufigkeit wird zwar als ein ideales Kriterium für die Aufnahme von Phraseologismen in Wörterbücher dargestellt, deren Ermittlung ist aber sehr schwierig, denn sie ist nur durch sehr aufwändige (und teure) Informantenbefragungen möglich (vgl. Sajankova in Vorb. zur Bestimmung der Geläufigkeit von Phraseologismen, sowie Baur/Chlosta (1996) und Durčo (in Vorb.) zur Methodik bei der Bestimmung von Minima für Sprichwörter).

DieKombinationvonFrequenzanalysenundGeläufigkeitsbestimmungen könnte eine Methodik ergeben, mit der sich phraseologische Optima (vgl. Hessky 1997a und 1997b in Hinblick auf den DaF-Unterricht) als Grundlage für phraseographische Arbeiten bestimmen ließen.

\subsection{2. Überprüfung lexikographischer Informationen durch die Analyse von Korpusbelegen}

Die Vermutung, dass die Variationsvielfalt von Phraseologismen stärker ausgeprägt ist, als bisher in der Forschung angenommen wurde, soll am Beispiel des Phraseologismus grünes Licht geben (Nennform in DUDEN 11, vgl. Drosdowski/Scholze-Stubenrecht 1998: 453) untermauert werden. In meiner Analyse von gut 100 Belegstellen in Deutscher Wortschatz wurden folgende, hier mit den Stellvertretern $j d$ und etw vereinfacht dargestellten Verwendungsformen des Phraseologismus gefunden:

(1) grünes Licht für jdn/etw

(2) jd/etw gibt/erteilt/signalisiert (jdm/etw) grünes Licht (für/zu etw)

(3) jd/etw erhält/bekommt/erwartet/braucht/erhofft sich/holt sich grünes Licht (für/zu etw) (von jdm) (mit etw)

(4) jd/etw hat grünes Licht

(5) etw bedeutet grünes Licht für jdn/zu etw

Negierte Formen sind möglich:

(6) Ohne einen konkreten Vorschlag zur so genannten Gegenfinanzierung gibt sie kein grünes Licht. 
(7) Noch hat der Vorstand von PKN aber offenbar kein grünes Licht für die Übernahme der Leuna-Anteile bekommen.

(8) Die Marzahner CDU-Fraktion - entscheidend für die Stimmenmehrheit - konnte auch nach einer Probeabstimmung kein grünes Licht für Ungar signalisieren.

\section{Außerdem kommt der Phraseologismus dekliniert vor:}

(9) Zur Fusion mit Veba hieß es, dass in Kürze mit grünem Licht aus Brüssel gerechnet werde.

(10) Bei grünem Licht durch die Brüsseler Behörde stehe einem Verkauf des Pakets an einen beliebigen Interessenten jedoch nichts mehr im Wege, sagte Keitel.

(11) Diese Rechtsunsicherheit könnte der Bayerischen Hausbau unter Umständen noch heuer auch vor dem Verwaltungsgerichtshof zu grünem Licht für ihre Pläne verhelfen, fürchteten Otto und Pfaffmann.

Die Korpusbelege geben 'Bereiche der öffentlichen Verwaltung oder Institutionen, Politik und Wirtschaft' als bevorzugte semantische Umgebung für die Verwendung des Phraseologismus vor. Diese sollten bei der Formulierung der Bedeutungsangabe berücksichtigt werden:

(12) Das Projekt eines direktstrahlenden europäischen Rundfunktsatelliten Europesat hat grünes Licht von den Mitgliedstaaten der Betreibergesellschaft Eutelsat erhalten.

(13) Angesichts dieser Sachlage gab eine Zweidrittel-Mehrheit der Delegierten am Wochenende grünes Licht für die mehrstufige, umfangreiche Kampagne insgesamt, die noch sechs weitere Massenmailings mit bis zu 100.000 Adressen zu anderen Themen umfassen soll.

(14) Der Oberkonservator gibt dem Bauherrn grünes Licht für den Abriss der oberen Arkaden.

(15) Am Mittwoch hat der Daimler-Aufsichtsrat dem Vorstand grünes Licht für die Abschlussverhandlungen mit Bund und Ländern gegeben.

(16) Als im Februar 1978, zum Höhepunkt der Terroristenhatz, der neu in die Strafprozessordnung eingeführte Paragraph 111 der Polizei grünes Licht zur Einrichtung von Kontrollstellen und Massenüberprüfungen gab, atmete so manch Uniformierter auf.

Analysen von Korpusbelegen geben außerdem Auskunft über - häufig nicht kodifizierte - morpho-syntaktische Restriktionen und bevorzugte Konstruktionen. So ergibt sich erst durch die Analyse der Korpusbelege zur Suchform am Rande die Information, dass der Phrase- 
ologismus sich am Rande verstehen (Nennform in DUDEN 11, vgl. Drosdowski/Scholze-Stubenrecht 1998: 565) mit der Bedeutung 'etwas ist selbstverständlich' nur in der Präsens-Indikativform der 3. Person Singular und nur in einem Hauptsatz vorkommt, dem ein mit dass eingeleiteter Nebensatz als Subjekt vorangestellt ist:

(17) Dass derAutokonzern nicht begeistert war und keine Drehgenehmigung auf seinem Gelände erteilte, versteht sich am Rande.

(18) Dass "Amerikanismen" in Texten über moderne Musik häufiger vorkommen als bei Aschenputtel, versteht sich am Rande.

(19) Dass für jede Art von fester und flüssiger Nahrung gesorgt ist, versteht sich am Rande.

Die Methoden der Korpuslinguistik stellen ein unentbehrliches Werkzeug für Lexikographen dar. Eine korpusbasierte Überprüfung der lexikographischen Informationen ist unerlässlich für phraseographische Arbeiten, denn Wörterbücher kommen ohne empirische Daten aus der Sprachverwendung nicht aus, wenn sie als Hilfsmittel für dieselbe brauchbar sein sollen. Die Korpuslinguistik ermöglicht eine methodisch sinnvolle Kombination aus "top-down"-Analysen von Phraseologismen in Texten und "bottom-up"-Beschreibungen der Phraseologie im Sprachsystem (vgl. Wotjak 1992: 39). Kombiniert mit anderen Werkzeugen ermöglicht das Heranziehen korpusorientierter Methodik eine umfassende und detaillierte Beschreibung von Phraseologismen.

\section{Phraseographie}

\subsection{Phraseologie in Wörterbüchern}

Eine grundsätzliche Unterscheidung gilt es zu machen für die metaphraseographische Diskussion über die Darstellung von Phraseologismen in allgemeinsprachlichen Wörterbüchern einerseits und in speziellen Phraseologiewörterbüchern andererseits (vgl. Fleischer 1997: 233). Weiterhin wird unterschieden "zwischen ein- und zwei- oder mehrsprachigen Wörterbüchern" (Fleischer 1997: 233).

Die lexikographische Behandlung von Phraseologismen wird nach wie vor kritisch reflektiert, wobei die phraseologischen Spezialwörterbücher nicht selten negativer beurteilt werden als die allgemeinen ... "(Fleischer 1997: 234). 
Die metaphraseographische Diskussion, d. h. die Bewertung der Phraseologie in Wörterbüchern, geht einerseits aus "Werkstattberichten" mit Vorschlägen zu einer neuen und besseren lexikographischen Darbietung hervor (vgl. z. B. Cheon 1998 (deutsch-koreanische Phraseologie); Kempcke 1987 (deutsche Phraseologie); Korhonen 2003 (deutsch-finnische Phraseologie); Welker 2003 (deutsch-portugiesische Phraseologie)). Andererseits entsteht sie als Ergebnis der Auswertung eines oder mehrerer Wörterbücher, häufig in Relation zu den Bedürfnissen fremdsprachlicher Nutzer und mit dem Ziel, Defizite aufzudecken.

$\mathrm{Zu}$ Defiziten in der Phraseographie und zur Darstellung der Phraseologie im Wörterbuch gibt es zahlreiche theoretische Überlegungen, Wörterbuchanalysen und Verbesserungsvorschläge (vgl. z. B. Braasch 1988; Burger 1998: 168 ff.; Cheon 1998; Fleischer 1997: 233 ff.; Földes 1996; Kjær 1987; Kühn 1989; Moon 1999; Pilz 1995; Stantcheva 1999; Wotjak 2001; Zöfgen 1994). Arbeiten, die sich auf die deutsche Phraseologie beziehen, kommen überwiegend zu dem Ergebnis, dass die Aufarbeitung und die Beschreibung der Phraseologie in gedruckten Wörterbüchern bestenfalls als mangelhaft zu bewerten sind. Als Konsequenz dieses Ergebnisses fordert Hessky (1999: 239 ff.) ein Umdenken in der Phraseographie, um die Phraseologie "von dem Korsett lexikographischer Tradition zu befreien" (Hessky 1999: 240), in der phraseologiespezifische Eigenschaften nicht berücksichtigt werden. Bemängelt wird nicht nur die Bearbeitung der Phraseologie in Wörterbüchern, sondern auch die nach wie vor vorhandene Diskrepanz zwischen der Phraseologieforschung und der lexikographischen Praxis. Die Ergebnisse der Phraseologieforschung finden nur sehr zögerlich Anwendung in der Lexikographie, "ein wirklicher Durchbruch bei der Integration phraseologischer Forschungsergebnisse in die Wörterbücher" (Zöfgen 1994: 211) war vor zehn Jahren noch nicht erfolgt.

Die Ergebnisse der metaphraseographischen Arbeiten zeigen Defizite in fast allen Bereichen der lexikographischen Bearbeitung und Beschreibung von Phraseologismen in deutschsprachigen Wörterbüchern. Die Ergebnisse lassen darauf schließen, dass die Wörterbücher im Bezug auf Phraseologie eigentlich unbrauchbar sein müssten. Dem sei hier exemplarisch entgegengehalten, dass DUDEN 11 Redewendungen und Sprichwörtliche Redensarten in der zweiten, neu bearbeiteten Auflage von 2002 auf dem Markt ist, so dass phraseologische Wörterbü- 
104

cher trotz aller Mängel offensichtlich vielfach gekauft und gewiss auch benutzt werden.

Außer Frage steht aber, dass die Metaphraseographie mit Recht darauf hinweist, dass - auch wenn im Laufe der letzten zehn Jahre einige Forschungsresultate aus der Phraseologie den Weg in die Wörterbücher gefunden haben - in der Phraseographie noch ein erhebliches Verbesserungspotenzial vorhanden ist.

\subsection{Theoretische Phraseographie}

Es gibt zahlreiche allgemeine metaphraseographische Überlegungen zur Konzeption phraseologischer Wörterbücher (vgl. z. B. Burger 1998; Cheon 1998; Fellbaum et al. in Vorb.; Fleischer 1992; Hallsteinsdóttir im Druck und in Vorb. a+b; Hessky/Ettinger 1997; Jónsson 1998; Jónsson 2001a; Koller 1987; Matešić 1983; Pilz 1987). Außerdem gibt es viele Gestaltungs- und Verbesserungsvorschläge, die sich auf spezielle Aspekte der Phraseographie beziehen, z. B. die Datenbankkonzeption von Piirainen (2000), die Beschreibungsvorschläge von Wotjak (1992) und Mudersbach (1998), die Konzeption zweisprachiger phraseologischer Wörterbücher von Filipenko (2002) sowie Farøs (2004a) Überlegungen zum einsprachigen dänischen Internet-Idiomwörterbuch. Überlegungen aus der theoretischen Phraseographie bildeten die Grundlage für die Konzeption einer zweisprachigen deutsch-isländischen Phraseologiedatenbank, die in Hallsteinsdóttir (im Druck, in Vorb. $\mathrm{a}+\mathrm{b}$ ) beschrieben wird. Ein erstes Eingeständnis nach Erfahrungen aus der phraseographischen Arbeit an dieser Datenbank betrifft die Integration von Forschungsergebnissen der Phraseologie in die phraseographische Arbeit. Die Forschung hat in vielen Bereichen, die für die Phraseographie relevant sind, nur wenige Ergebnisse geliefert. Die Grundlage für eine Integration ist in diesen Bereichen also noch nicht vorhanden. Dies betrifft vor allem die korpusgestützte Analyse und die darauf aufbauende umfassende Beschreibung einzelner Phraseologismen. Solche Daten sind, bis auf Bedeutungsbeschreibungen, meist nur sehr lückenhaft vorhanden.

Es gibt durchaus Wörterbücher für die Rezeption von Phraseologismen, die unterschiedlich detaillierte Bedeutungsangaben anbieten. Diese Wörterbücher spiegeln in gewissem Sinne den Stand der Forschung wieder, denn die Beschaffenheit der phraseologischen Bedeutung stellt einen der bisher am ausführlichsten diskutierten Aspekten 
der Phraseologie dar. Außerdem ist eine Bedeutungsbeschreibung, die das Verstehen eines Phraseologismus sichern soll, eine relativ "einfache" Aufgabe, verglichen mit der Komplexität einer Beschreibung, die zum richtigen Gebrauch eines Phraseologismus führen soll. Eine verwendungsbezogene Beschreibung muss eine genaue Bedeutungsbeschreibung mit einschließen, deshalb wird hier die metalexikographische Schlussfolgerung daraus gezogen, dass ein adäquat konzipiertes Phraseologiewörterbuch für den aktiven Phraseologiegebrauch ein spezielles Wörterbuch für das Verstehen (die passive Verwendung) von Phraseologismen überflüssig machen könnte.

Ein nach wie vor heterogenes Gebiet in der Phraseographie sind pragmatische Angaben. Über pragmatische Angaben zu Phraseologismen sind viele Theorien aufgestellt worden, und die Forderung nach "aussagekräftigen pragmatischen Kommentaren" (Kühn 1994: 422) als die "Rekonstruktion von Gebrauchsregeln, nach denen Phraseologismen verwendet werden" (Kühn 1994: 426) - in verschiedenen Ausprägungen an Stelle traditioneller Stilmarkierungen wird immer wieder gestellt (vgl. Kühn 1994: 422 ff.) Wie dieser Kommentar konkret aussehen soll, damit ein Sprecher den betreffenden Phraseologismus nicht nur versteht, sondern auch adäquat anwenden kann, dafür gibt es nur wenige Beispiele.

Ich möchte für einen funktionalen Beschreibungsansatz in Anlehnung an die Laswell-Formel plädieren (vgl. auch "die pragmatischen Verwendungsbestimmungen (wer, wann, zu welchem Zweck, bei welchem Medium usw.) ...” (Götz 1977: 98 [Götz, D. (1977): Idiomatik. In: K. Schröder und Th. Finkenstaedt (Hg.): Reallexikon der englischen Fachdidaktik. Darmstadt, 96-99] - zitiert nach Glaap 1979: 487)), mit dem auch Verwendungsrestriktionen und bevorzugte semantisch-pragmatische Verwendungsumgebungen erfasst und beschrieben werden können. Ob die Aspekte der Pragmatik als integrierter Bestandteil der Bedeutungserklärung (als eine Art semantischer Mehrwert, vgl. z.B. Dobrovol'skij 2002 und Kühn 1994) beschrieben oder in speziellen pragmatischen Kommentaren als Gebrauchsanleitungen erfasst werden, sollte je nach Benutzergruppen und Wörterbuchzweck entschieden werden.

Die Computerphraseographie bietet auch schon die Möglichkeit eines onomasiologisch-situativen Zugriffs auf die Phraseologismen im 
Wörterbuch. Eine entsprechende Markierung der Bedeutung und der Verwendungssituation würde das Abrufen aller Phraseologismen ermöglichen, die in einer vorgegebenen Situation verwendbar sind. Dies ist vor allem bei intersprachlichen Vergleichen von asymmetrischen Phraseologiegruppen relevant, vgl. Tabelle 1:

\begin{tabular}{|l|l|l|l|l|}
\hline Verwendung & $\begin{array}{l}\text { Begrüßung am } \\
\text { Morgen }\end{array}$ & $\begin{array}{l}\text { Begrüßung am } \\
\text { Tag }\end{array}$ & $\begin{array}{l}\text { Begrüßung am } \\
\text { Abend }\end{array}$ & $\begin{array}{l}\text { Verabschiedung } \\
\text { am späten } \\
\text { Abend bzw. } \\
\text { Wunsch beim } \\
\text { Schlafengehen }\end{array}$ \\
\hline Deutsch & guten Morgen & guten Tag & guten Abend & gute Nacht \\
\hline Isländisch & \multicolumn{2}{|c|}{ góðan dag } & gott kvöld & góða nótt \\
\hline Dänisch & godmorgen & goddag & godaften & godnat \\
\hline
\end{tabular}

Tabelle 1: Beispiele für Asymmetrie in der Verwendung kongruenter Phraseologismen.

Im Deutschen hat guten Morgen bzw. godmorgen im Dänischen (die alte Form god morgen ist nicht mehr gebräuchlich, daher ist die BegrüBungsformel im Dänischen keine Mehrwortverbindung mehr) die Verwendung 'eine Begrüßung am Morgen'. Im Isländischen gibt es dafür keinen kongruenten Phraseologismus, der situativ adäquate Phraseologismus ist góðan dag, der einen breiteren Verwendungsrahmen hat als die deutschen und dänischen Kongruenten guten Tag und goddag. Die Aufstellung hier verdeutlicht allerdings nur die Asymmetrie innerhalb dieser Gruppe, sie sagt noch nichts darüber aus, welche Relationen zu nicht-phraseologischen sprachlichen Einheiten oder zu anderen Phraseologismen bestehen, die z. B. (a) in denselben Verwendungssituationen möglich sind, wie z. B. Grüß Gott oder hej (med dig) oder (b) dieselbe/ähnliche Form beinhalten, wie ha' en (rigtig/fortsat) god dag / einen schönen Tag noch.

Die Beschreibung und Darstellung morphosyntaktischer, semantischer und funktional-pragmatischer Eigenschaften von Phraseologismen sind theoretisch sehr heterogen. Es gibt sehr viele detaillierte Beschreibungsmodelle für einzelne Aspekte, die jedoch kaum die mehrdimensionalen Möglichkeiten der Computerphraseographie mit einbeziehen. Diese Möglichkeiten in einem kontrastiven, mehrsprachigen Phraseologiewörterbuch unter funktionalem Aspekt (vgl. z. B. Farø in Vorb. b) zu 
realisieren, in dem benutzerspezifische Bedürfnisse mehrerer Benutzergruppen berücksichtigt werden (vgl. Bergenholtz 1997), ist eine wichtige Aufgabe der Phraseographie.

\subsection{Zwei- und mehrsprachige Phraseographie}

Bei der zwei- und mehrsprachigen Phraseographie müssen zuerst die Begriffe zweisprachiges Wörterbuch und mehrsprachiges Wörterbuch erklärt werden (vgl. ausführlich zur Frage, "was ist ein bilinguales Wörterbuch?" Tarp 2004). In zweisprachigen Phraseologiewörterbüchern (mehrsprachige Phraseologiewörterbücher sind noch sehr selten und meist in Form von einfachen Listen äquivalenter Phraseologismen, z. B. Jensen 1982) handelt es sich in der Regel um unidirektionale Wörterbücher, in denen Phraseologismen in einer Objektsprache (auch: Ausgangssprache) mit Hilfe der anderen Sprache auf einer Metaebene beschrieben werden, wobei Phraseologismen der Metasprache (auch: Zielsprache) nur der Erklärung der Objektsprache (meist durch die Angabe von möglichen Äquivalenten) dienen. In diesem Zusammenhang wird hier von den gängigen Termini Ausgangssprache und Zielsprache für Wörterbuchinhalte Abstand genommen. Diese sind durch die Übersetzungswissenschaft sehr stark von der Vorstellung geprägt, dass etwas aus einer Sprache in eine andere Sprache übertragen wird. In Wörterbüchern mit mehr als einer Objektsprache muss der Transfer von Informationen in beide Richtungen stattfinden.

Als ein Beispiel für ein unidirektionales zweisprachiges Wörterbuch (vgl. z. B. die Diskussion über phraseologische Übersetzungswörterbücher in Kromann 1987) soll das Konzept von Dobrovol'skij/Filipenko (2003) dienen, in dem deutsche Idiome (Deutsch als Objektsprache) auf Russisch (als Metasprache) erklärt werden. Das Wörterbuch ist nur für russischsprachige Benutzer interessant, die sich über die deutsche Phraseologie informieren wollen. Ihre Benutzungsbedürfnisse werden für sowohl den passiven (Verstehen) als auch den aktiven (Verwenden, Übersetzen) Gebrauch von deutschen Idiomen auf hohem Niveau erfüllt, für die im Wörterbuch vorkommenden russischen Phraseologismen erfährt der Benutzer jedoch nur, für welche deutsche Idiome sie als Äquivalente in Frage kommen. Um als bidirektionales Wörterbuch fungieren zu können, fehlen adäquate Erklärungen und Verwendungsbeispiele für die russischen Idiome. 
Nun sind die Konzeptionen zweisprachiger unidirektionaler Wörterbücher in der Regel am gedruckten Wörterbuch orientiert, dessen Zweidimensionalität die Konzeption eines bidirektionalen Wörterbuchs zugegebenermaßen schwierig macht. Für kleine Sprachen ebenso wie für viele spezielle Fachgebiete ist es, finanziell und vom Arbeitsaufwand her, fast unmöglich, an dem Prinzip der Unidirektionalität mit aktiven und passiven Wörterbüchern festzuhalten. (Die Unidirektionalität eröffnet allerdings die Möglichkeit, für jedes Sprachenpaar vier (ein aktives und ein passives Wörterbuch für jede Muttersprache) statt einem Wörterbuch zu verkaufen - ein "Ökonomieprinzip", das nicht nur die "Bedürfnisse der Wörterbuchbenutzer (...) mit einem Minimum von lexikographischem Aufwand ein Maximum an lexikographischen Informationen" (Kromann 1987: 184) berücksichtigen will).

Es ist die Aufgabe der Phraseographie, ein mehrsprachiges Wörterbuchkonzept für bidirektionale (und somit polyfunktionale, vgl. Bergenholtz 1997) elektronische Wörterbücher zu schaffen, in denen jede Sprache als Objektsprache und als Beschreibungssprache (paarweise Beschreibung der Sprachen) dient. Ein solches Konzept setzt allerdings voraus, dass sich auch die interlinguale Phraseologieforschung nicht nur als "Einbahnstraße" (Hessky 1997a: 258) betrieben wird, sondern dass kontrastive Arbeiten von beiden Sprachen ausgehen müssen.

\subsection{Die Äquivalenzproblematik}

Eine Arbeit über Phraseographie, die sich auch mit zwei- und mehrsprachiger Phraseographie befasst, kommt an dem Begriff Äquivalenz nicht vorbei. In einem zwei- oder mehrsprachigen Wörterbuch ist anzunehmen, dass ein Benutzer nicht nur Informationen zur Bedeutung oder zu morphosyntaktischen und pragmatischen Verwendungsmöglichkeiten sucht. Diese können u. U. auch ohne Einbeziehung der Äquivalenz beschrieben werden. Der Benutzer wird aber in der Regel auch erwarten, Antworten darauf zu finden, welche Phraseologismen als mögliche Übersetzungen in Frage kommen. Es ist also notwendig, potentiell äquivalente Phraseologismen kontrastiv zu erfassen und phraseographisch $\mathrm{zu}$ beschreiben (die Typologisierung von Äquivalenzbeziehungen wird z. T. auch mit ihrer Relevanz für die Phraseographie begründet (vgl. die Diskussion der Äquivalenz in Dobrovol'skij 1997b: 46ff.)). 
Inzwischen gibt es eine unüberschaubare Anzahl an Arbeiten zur Äquivalenz von Phraseologismen, die fast alle eigene Äquivalenzmodelle bzw. Äquivalenztypen (vgl. z. B. die Diskussion von Äquivalenztypologien in Eismann 1995: 97ff.) anbieten. Diese berücksichtigen in der Regel einen oder mehrere Aspekte wie "Bildhaftigkeit, Semantik, Lexik, Syntax und Pragmatik" (Korhonen 1991: 123; vgl. auch Wotjak 1992b), die meist isoliert auf der Ebene des Sprachsystems verglichen werden. Aber auch weitere Aspekte wie z. B. die Verwendung von Phraseologismen in Texten, Übersetzung von Phraseologismen und Bedürfnisse der Phraseodidaktik gehen in die Äquivalenzforschung ein (vgl. z.B. ausführlich zur Äquivalenzproblematik in Hessky 1987 und zur Idiomäquivalenz in Farø in Vorb. b+c; sowie die Darstellungen in Hessky 1997a; Holzinger 1998; Kim-Werner 1998; Koller 1994: 356 ff.; Korhonen 1991).

Dobrovol'skij (1997b) stellt nach der Analyse der kontrastiven Phraseologieforschung fest, dass "die eigentlich lexikographischen Fragestellungen bis auf einige Ausnahmen kaum Diskussionsgegenstand waren" (Dobrovolj'skij 1997b: 46).

Aus der Spezifik der kontrastiven Linguistik ergibt sich die Relevanz
dieser Richtung für die zweisprachige Lexikographie. In bezug auf
die Idiom-Komponente des Lexikons bedeutet dies, daß die kontrastiv
orientierten phraseologischen Konzeptionen eine theoretische Grund-
lage für die Entwicklung zweisprachiger Phraseologie-Wörterbücher
verschiedener Typen liefern sollen. (Dobrovol'skij 1997b: 45-46)

In der Praxis ist es aber nicht ungewöhnlich, dass die Darstellung von Phraseologismen in Wörterbüchern die Grundlage für Äquivalenzuntersuchungen bildet. Wenn die lexikographische Beschreibung der Phraseologie schon mangelhaft ist, diese aber als Grundlage für Äquivalenzuntersuchungen verwendet wird, die wiederum als Grundlage für phraseologische Wörterbücher dient, dann beißt sich die Katze doch in den Schwanz. Die Ergebnisse solcher Vorgehensweisen sind bestenfalls als fragwürdig anzusehen.

Spätestens mit dem Einzug der Korpuslinguistik in die Phraseologieforschung begann die von Wotjak (1992: 40) vorhergesagte Erweiterung der kontrastiven Phraseologie "in Richtung Sprachverwendung" (Wotjak 1992: 40). Damit wird eine Aufhebung der traditionellen, „konsequenzreichen Einschränkung" (Koller 1994: 358) der kontrastiven Linguistik auf das Vergleichen von isolierten Phraseologismen ermöglicht. Es gilt 
die schon bei Häusermann angestrebte Perspektive, die oben mit Beispielen untermauert wurde: "[g]enau erfassbar sind Frasmen, wie gesagt, nicht in der langue, sonder nur in der parole." (Häusermann 1977: 104). Der Blick wird auf den Sprachgebrauch gerichtet und eine funktionale Beschreibung (vgl. Farø in Vorb. $\mathrm{a}+\mathrm{b}$ ) von Phraseologismen in Texten wird in den Vordergrund gestellt. Im Zusammenhang mit der Phraseographie muss dabei die Brauchbarkeit der Äquivalente für den aktiven Sprachgebrauch (bzw. das Übersetzen) im Mittelpunkt stehen und nicht der Vergleich von Phraseologismen im Ausgangstext und Phraseologismen als Resultat von Übersetzungen im Zieltext.

Dobrovol'skij betont die Wichtigkeit, dass man nur dann von einer Gleichwertigkeit (i. e. Äquivalenz) sprechen kann, wenn ein Phraseologismus in der gleichen Situation in der L1 und der L2 verwendet werden kann.

Vom Standpunkt des Wörterbuchbenutzers aus ist es jedoch völlig unwichtig, ob ein Idiom der Ausgangssprache L1 in die Zielsprache L2 mit einem absoluten (1), einem partiellen (2) oder einem nichtidiomatischen Äquivalent (3) übersetzt wird. Wichtig ist nur der Grad der funktionalen Äquivalenz, d.h. wenn der Benutzer die L2-Übersetzung in den gleichen funktionalen Domänen wie die entsprechende L1-Einheit gebrauchen kann, erfüllt der betreffende Wörterbuchartikel seine Rolle. (Dobrovol'skij 1997b: 47)

Eine Fokussierung auf funktionale Äquivalenz führt zu dem Problem, dass viele Phraseologismen im Sprachsystem eine vage Bedeutung haben, die erst im konkreten Text festgelegt wird. Wenn man von einer funktionalen Äquivalenz (auch Farø (in Vorb. b) bezieht sich auf die "Funktion im Text" als Grundlage für die Übersetzung von Phraseologismen) ausgeht, dann kann ein äquivalenter Phraseologismus jeweils nur im Bezug auf konkrete Texte bestimmt werden, die Äquivalenz wäre somit als Eigenschaft situativ einmalig. Auch Koller sieht die "Übersetzungsäquivalenz nicht auf der Ebene der Bedeutung, sondern der Bezeichnung" (Koller 1994: 362)), und er weist darauf hin,

... daß im Textzusammenhang meistens keineswegs alle, sondern nur bestimmte übersetzungsrelevante Bedeutungsdimensionen wiedergegeben werden müssen. (Koller 1994: 362)

In der Phraseographie gilt es also, diese Eigenschaft der phraseologischen Bedeutung (vgl. z. B. Hallsteinsdóttir 2001 zur Beschaffenheit der phraseologischen Bedeutung) zu berücksichtigen. Eismann plädiert 
in diesem Zusammenhang dafür, Äquivalenzangaben im Wörterbuch durch eine ausführliche "erklärende Umschreibung der Bedeutung und der Gebrauchsbedingungen der Phraseologismen" zu ersetzen, "der allenfalls die Angabe einer Entsprechung hinzugefügt werden sollte" (Eismann 1995: 95). Das Problem der Bedeutungsvagheit soll durch eine ausführliche Beschreibung des Phraseologismus minimiert werden:

... es müssen neben der wörtlichen Übersetzung und der Beschreibung der Bedeutung und allfälligen Erläuterungen der Gebrauchsbedingungen (samt sämtlichen Restriktionen) Konnotationen und usuellen Verwendungsweisen (hier sind Beispiele eine große Hilfe) angegeben werden. In diesem Bereich kann und muß daher die einsprachige Phraseologie die Basis liefern. Zweisprachige Wörterbücher sollten auf dieser Basis, aber nur in intensiver Zusammenarbeit kompententer Sprecher jeweils beider Sprachen, erstellt werden. (Eismann 1995: 115)

Wenn eine solche Beschreibung vorliegt, dann kann davon ausgegangen werden, dass für die phraseographische Erfassung der funktionalen Äquivalenz für die normale Verwendung von Phraseologismen eine Äquivalenztypologie ausreicht, die sich auf die phraseologische Bedeutung bezieht (die inhaltliche Äquivalenz, vgl. Korhonen 1991: 123). Die Form eines Phraseologismus spielt nur sekundär eine Rolle. Funktionale Äquivalente, die außerdem kongruent sind, werden ja auch über die inhaltliche Äquivalenz erfasst. Diese Erfassung ist allerdings wichtig im Zusammenhang mit der "thematisierten Verwendung" von Phraseologismen (vgl. Farø in Vorb. b). Eismanns Vorschlag soll nur hinzugefügt werden, dass die Bedeutungsvarianten in der Regel durch Korpusbelege erfasst und beschrieben werden können. Somit gibt es die Möglichkeit, Verwendungsbeispiele für potentielle phraseologische Äquivalente in adäquaten Kontexten in beiden Sprachen anzugeben.

Im Wörterbuch sollte die Angabe eines phraseologischen inhaltlichen Äquivalents darüber hinaus durch einen Äquivalenzkommentar ergänzt werden, der die Äquivalenzbeziehung unter Einbeziehung anderer Aspekte genauer erläutert (vgl. z. B. die Vorschläge in Dobrovol'skij 1997b: 53 ff.) Bei potentiellen falschen Freunden (vgl. z. B. Piirainen 1999 zur Problematik der falschen Freunde) ist es außerdem didaktisch sinnvoll, explizit auf die nicht vorhandene Äquivalenz hinzuweisen, um Interferenzen aus der Muttersprache zu verhindern. Muttersprachlicher Transfer bei inhaltlich äquivalenten Kongruenten sollte auch beschrie- 
ben werden, damit die Bestätigung vorhandener Äquivalenz gegeben und "Spiegelübersetzungen" (Hessky 1997a: 258) verhindert werden (vgl. Hessky (1997a: 257) zu den Termini Transfer als positiver und Interferenz als negativer Einfluss der Muttersprache). Außerdem kann nicht davon ausgegangen werden, dass den Wörterbuchbenutzern die im Sprachsystem vorhandenen muttersprachlichen Äquivalente bekannt sind (vgl. z. B. Hallsteinsdóttir 2001).

Als einen wichtigen Faktor der Äquivalenzbestimmung von Phraseologismen nennt Dobrovol'skij weiterhin den Geläufigkeitsgrad, denn Totaläquivalente im Sprachsystem zweier Sprachen müssen nicht gleichwertig in der Sprachverwendung vorkommen (vgl. die Darstellung oben).

Einen weiteren wichtigen Faktor der funktonalen Äquivalenz stellt die Identität der inneren Form dar. Die Bildlichkeit ist eine wichtige Komponente des Inhaltsplanes jeder motivierbaren Lexikoneinheit, deshalb muß die innere Form bei der Übersetzung mit berücksichtigt werden. Andernfalls verändert sich das assoziative Umfeld des betreffenden Idioms. (Dobrovol'skij 1997b: 47)

Farø (in Vorb. b) hat jedoch festgestellt, dass die überwiegende Anzahl der 1.200 Ausgangsphraseologismen in seinem Korpus mit deutschen und dänischen Idiomen in literarischen Texten und deren Übersetzungen wohl wie ganz normale lexikalische Einheiten ohne spezielle Intentionen, Markierungen oder Berücksichtigung der Bildsphäre verwendet werden. Ein wichtiges Ergebnis seiner Untersuchung betrifft die Rolle der Kulturspezifik in der Phraseologie. Es wird in der Phraseologieforschung häufig davon ausgegangen, dass das Vorhandensein von Kulturspezifik in der Phraseologie ein Faktum darstellt (vgl. die Diskussion über die undifferenzierte Verwendung des Terminus Kulturspezifik in der Phraseologie in Dobrovol'skij (1999: 41 ff.) und Farø (in Vorb. $\mathrm{a}+\mathrm{b}$ )). Farøs Resultat ist, dass eine Kulturspezifik in der deutsch-dänischen Phraseologie kaum vorkommt. Er bestätigt damit Dobrovol'skijs Annahme, dass fehlende Äquivalente in der Phraseologie

nicht auf ihre kulturelle Spezifik, sondern auf Unterschiede in der Versprachlichung bestimmter Entitäten mit Mitteln der sekundären Nomination zurück[gehen]. (Dobrovol'skij 1999: 42) 
Phraseologische Bilder sind demnach einfach "sprachliche Zufälle" (Dobrovol'skij 1999: 49 - vgl. auch seine Differenzierung zwischen "Kognitivem und Kulturellem" 1999: 53).

Die zwei Eigenschaften Kulturspezifik und Bildlichkeit sind die im Sprachunterricht (in der Muttersprache und in Fremdsprachen) am häufigsten didaktisierten Eigenschaften der Phraseologie (vgl. Kühn 1993 zur "Funktionalisierung der Phraseodidaktik auf Sprach- und Kulturgeschichte" (Kühn 1993: 61) einerseits und "auf Bildlichkeit und Anschaulichkeit" (Kühn 1993: 68) andererseits (vgl. dazu auch Kühn 1992). Sie stellen sicherlich auch eine Hilfe beim Erlernen der Phraseologie einer Sprache dar, für die Phraseographie sind sie auch im Bezug auf die Phraseodidaktik von Relevanz.

Es deutet einiges darauf hin, dass die "Doppelnatur" (Häusermann 1977: 11) von Phraseologismen, d.h. phraseologiespezifische Eigenschaften wie Bildsphäre, komplexe Semantik, Polylexikalität oder morphosyntaktische Besonderheiten in der Sprachverwendung nur eine untergeordnete Rolle spielen:

- Phraseologische Bilder werden nur in thematisierter (vgl. Farø in Vorb. b) "sprachspielerischer" Verwendung von Phraseologismen aktiviert. In der Forschung sind zwar viele Untersuchungen zur thematisierten Verwendung durchgeführt worden, vermutlich handelt es sich hier aber nur um eine kleine Anzahl des tatsächlichen Vorkommens an Phraseologismen im Sprachgebrauch $(\approx 10 \%)$.

- Historisch-etymologisches Wissen über Phraseologismen wird ebenfalls nur dann aktiviert, wenn dies für das Verstehen relevant ist.

Es ist falsch zu glauben, beim Sprechen/Schreiben, Lesen/Hören reflektiere man ständig bewußt die Etymologie und Kulturgeschichte, die an Redensarten hängt. (Kühn 1993: 70)

- Auch wenn potentiell eine nicht-phraseologische Konstruktion der einzelnen Komponenten möglich ist, kommt diese in der Sprachverwendung vielen Fällen kaum vor, denn die dominante phraseologische Bedeutung (vgl. Giora 1997 zur Theorie der dominanten Bedeutung) verdrängt eine Verwendung der Komponenten als freie Wortverbindung, vgl. jemand bekommt grünes Licht/gelbes Licht/ rotes Licht weiter unten.

Schon Häusermann hat darauf hingewiesen, dass es für den "inkompetenten Sprecher" (Häusermann 1977: 119) an sich keinen prinzipiellen 
Unterschied zwischen freien Wortverbindungen und Phraseologismen gibt, die primäre Funktion ist bei beiden die Benennungsfunktion (vgl. zur "Frage der Äquivalenz von Frasmus und Wort" bei Häusermann 1977: 11 ff.)

Unter Grammatik verstehen wir Regeln zum Kombinieren von Einheiten. Die Bedeutung ist der Grund dafür, dass sie kombiniert werden. Wenn wir einen solchen Begriff der Bedeutung annehmen wollen, unterscheiden sich die phraseologische und die lexikalische Bedeutung nicht grundsätzlich: sie beide gewährleisten Sprache. Aber die Bedeutung in der Phraseologie ist vager: unter Umständen regt sie die Phantasie des Sprechers / Hörers an, es kann dazu- oder weggedacht werden. Notwendig ist dies jedoch nicht. (Häusermann 1977: 112)

Der "inkompetente Sprecher" verfügt auch über eine sprachliche "Common-Sense-Kompetenz" (vgl. Feilke 1993: 7), die ihn "wörtliche" Bedeutungen und sprachliche Bilder nur dann wahrnehmen lässt, wenn sie von der dominanten sprachlichen Norm - die ja nicht unbedingt "wörtlich" ist (vgl. Giora 1997) - abweichen.

$\mathrm{Zu}$ wenig beachtete Aspekte der Phraseologie sind intrasprachliche Relationen von Phraseologismen zu anderen Phraseologismen einerseits und zu nicht-phraseologischen sprachlichen Einheiten andererseits. Die Beschreibung dieser Relationen ist sehr wichtig für die Beschreibung intersprachlicher Relationen. Als Beispiel für intrasprachliche Relationen soll hier wieder jd bekommt grünes Licht angeführt werden:

(20) Mal gaben demokratische Präsidenten grünes Licht, dann schalteten konservative wie zuletzt George W. Bush auf Rot.

(21) Unterdessen sagte US-Außenamtssprecher John Dinger in Washington, Palästinenser-Präsident Yasser Arafat müsse dafür sorgen, daß es für Selbstmordanschläge wie in Tel Aviv nicht nur "kein grünes Licht", sondern "rotes Licht" gebe.

In Relation zu diesem Phraseologismus (dieselbe Bildsphäre bzw. kognitive Domäne, die in den Beispielen (20) und (21) thematisiert wird) werden auch jd bekommt rotes Licht und (viel seltener) jd bekommt gelbes Licht verwendet. Die Häufigkeit der Verwendung ist aber nicht gleichwertig mit der Verwendung von jd bekommt grünes Licht. In einem Wörterbuch muss auf jeden Fall darauf hingewiesen werden, dass es diese Phraseologismen gibt, und dass sie viel seltener vorkommen als eine nicht-phraseologische Bedeutung der Wortkombination. Von 48 
Belegen für gelbes Licht in Deutscher Wortschatz enthält nur einer den Phraseologismus:

(22) Die USA, die Israel damals nach Ansicht vieler Beobachter zumindest gelbes Licht gegeben hatten, sollten diesen Fehler nicht wiederholen.

Und von 164 Belegen für rotes Licht enthalten nur 20 den Phraseologismus:

(23) Nur ein einziges Mal im Verkaufsrekordjahr 1986 gab es rotes Licht von der Berliner Behörde.

(24) Im Hause Klemann hatte man sich schon auf eine öffentliche Präsentation von Entwürfen vorbereitet, aus Bonn kam rotes Licht.

Dieses Verhältnis zwischen der phraseologischen und der nicht-phraseologischen Bedeutung ist umgekehrt bei grünes Licht. Bei den 256 analysierten Belegen kommt nur dreimal eine nicht-phraseologische Bedeutung vor, d. h. hier dominiert eindeutig die phraseologische Bedeutung der Wortkombination. Für die zweisprachige Phraseographie muss geklärt werden, ob die Phraseologierelationen einer anderen Sprache die gleichen Verwendungsmuster aufweisen.

Im interlingualen Vergleich ist eine Beschreibung phraseologischer Gruppen dann besonders wichtig, wenn es eine intralinguale Asymmetrie innerhalb der Gruppen gibt, wie bei folgenden kongruenten Phraseologismenpaaren, die im Deutschen und im Dänischen dieselben Bedeutungsbeziehungen, Antonymie sowohl auf der nicht-phraseologischen als auch der phraseologischen Ebene, aufweisen, im Isländischen jedoch, trotz der Antonymie auf der nicht-phraseologischen Ebene der Komponenten, keine Antonymiebeziehung zwischen den Phraseologismen vorhanden ist:

(25) Deutsch: das Gesicht verlieren 'etwas von seiner Geltung einbüßen, sein Ansehen verlieren' - das Gesicht wahren 'so tun als ob alles in Ordnung sei' (vgl. DUDEN 11);

(26) Dänisch: tabe ansigt 'sein Ansehen verlieren'- redde ansigt 'den Verlust seines Ansehens vermeiden' (vgl. Andersen 2001: 26)

(27) Isländisch: missa andlitið 'sichtbar sehr überrascht werden' - bjarga/ halda andlitinu 'nach außen hin normal wirken, so tun als ob alles in Ordnung sei'

Die Erforschung dessen, inwieweit andere phraseologische Relationen als die bekannten falschen Freunde das Verstehen und die Verwendung von Phraseologismen beeinflussen, steckt noch in den Kinderschuhen. 
Die Ergebnisse einer ersten Untersuchung für Deutsch als Fremdsprache mit der Beschreibung möglicher phraseologischer Relationen werden in Hallsteinsdóttir (2001) dargestellt.

Wenn nun die Bedeutung die Vergleichsgrundlage bildet und die Form für die Äquivalenzbestimmung a priori keine Rolle spielt (wohl aber für die Erfassung von Kongruenten wie den falschen Freunden), dann gibt es wahrscheinlich nur in seltenen Fällen eine Nulläquivalenz. Ein Verzicht auf das Formkriterium eröffnet außerdem die Möglichkeit für die Angabe nicht-phraseologischer Äquivalente, womit auch eine wichtige Einschränkung der bisherigen Äquivalenzforschung aufgehoben wäre. Eine solche Herangehensweise ist eine erste Voraussetzung für die Erfassung von Wortschatzrelationen und sie ermöglicht die Integration der Phraseologie in Wörterbuchprojekte, die sich auf bestimmte Themenbereiche beziehen (vgl. z. B. Jesenseks mehrsprachiges phraseologisches Lehrwerk (Jesensek in Vorb.) und die Lexin-Projekte in den skandinavischen Sprachen (für Isländisch: www.lexis.hi.is/lexin_ny.html, für Dänisch: http://lexin.vhostip5.isop. net.uni-c.dk/ für Norwegisch: http://decentius.hit.uib.no/lexin.html?uilang=nbo und für Schwedisch: www-lexikon.nada.kth.se/skolverket/ lexin.shtml).

\section{Phraseographie und Phraseodidaktik}

Die Integration von Übungen zur Phraseologie in phraseologische Nachschlagewerke bzw. Übungsbücher mit Wörterbuchcharakter für das Erlernen der Phraseologie (vgl. z. B. Herzog et al. 1976) haben eine relativ lange Tradition (vgl. die Darstellung in Ettinger 1998: 202 ff. und als Beispiel für die Umsetzung des phraseodidaktischen Dreischrittes im Wörter- und Übungsbuch von Hessky/Ettinger 1997). Die Phraseodidaktik (vgl. Kühn 1992) stellt dagegen ein relativ junges (erste Arbeiten ca. 1975 - Ettinger (1998: 205) nennt als 'revolutionären' Vorreiter die Arbeit von D. Götz (1976): Stilistik und Idiomatik im Englischunterricht) und heterogenes Forschungsgebiet dar (vgl. Wotjak 1996), in dem die Phraseographie allerdings meist nur am Rande eine Rolle spielt. Einen kurzen Überblick über das Forschungsgebiet der Phraseodidaktik, als "de[n] Teilbereich der Phraseologie, der sich mit der systematischen Vermittlung von Phraseologismen im mutter- oder 
fremdsprachlichen Unterricht befaßt" (Ettinger 2001: 88), bietet Ettinger (2001).

Wörterbücher spielen in der Phraseodidaktik nur eine untergeordnete Rolle. Zwar wird in phraseodidaktischen Arbeiten manchmal auf die Möglichkeit hingewiesen, dass man „die Arbeit mit dem Wörterbuch mit Phraseologismen trainieren" könne (Schreiter 1996: 56; vgl. auch Korpi-Käkelä 1996: 47 ff.) oder es wird die "wichtige Rolle" der Wörterbuchbenutzung "[b]eim Verstehen, Reflektieren, Erlernen, Üben und Anwenden von phraseologischen Redewendungen" (Földes 1996: 64) betont. Eine Beschreibung der Integration der Wörterbücher in den Unterricht und die Didaktisierung der Wörterbuchinhalte einerseits und Anleitungen "zur effektiven Nutzung phraseologischer Wörterbücher" (Kühn 1992: 170) andererseits fehlen aber in der Regel. Der erfolgreiche Umgang mit Phraseologie in Wörterbüchern wird nicht vermittelt.

Die primäre Aufgabe der Phraseodidaktik ist es, dem Lerner die Fähigkeit zu vermitteln, Phraseologismen als solche zu erkennen, die Bedeutung von Phraseologismen aus dem Kontext zu erschließen und "Phraseologismen situationsangemessen, adressatenspezifisch sowie textsortentypisch zu gebrauchen" (Kühn 1994: 413). Die Konsultation von Wörterbüchern sieht er nur in Ausnahmefällen vor (vgl. Kühn 1994: 425 und Kühn 1992: 180). Dies begründet er teils mit dem schlichten Nichtvorhandensein von adäquaten Wörterbüchern und teils mit Defiziten in den vorhandenen Phraseologiewörterbüchern. Sein Fazit ist,

... daß die bisherigen Wörterbücher zu Phraseologismen weder als

Nachschlagehilfen geeignet sind, noch dem genauen Verständnis von Bedeutungen der Phraseologismen dienen, geschweige denn zur Textproduktion eingesetzt werden können ... (Kühn 1992: 170)

Die allgemeine phraseodidaktische Auffassung ist, dass die Bedürfnisse der Sprachlerner in den Wörterbüchern nur ungenügend berücksichtigt werden (vgl. Babillon 2001: 105). Die natürliche Konsequenz in der Phraseographie wäre, die Erkenntnisse und die Bedürfnisse der Phraseodidaktik in Phraseologiewörterbüchern zu berücksichtigen.

Die phraseodidaktischen Wünsche in Bezug aufden Wörterbuchinhalt und die Aufarbeitung der Wörterbuchinformationen sind schon seit dem Erscheinen der Überlegungen zu idealen Phraseologiewörterbüchern von Koller (1987) und Pilz (1987) klar formuliert. Dazu gehören 
u. a. (vgl. Babillon 2001: 105-106; Ettinger 1998: 210 ff und 2001: 99100; Hessky/Ettinger 1997; Koller 1987: 111 ff.; Kühn 1993: 74; Pilz 1987):

- Informationen über Varianten (u. a. Negation und paradigmatische Austauschmöglichkeiten einzelner Komponenten) und Modifikationsmöglichkeiten.

- Die Erklärung morphosyntaktischer und semantischer Restriktionen sowie pragmatischer Verwendungsregeln.

- Umfassende Bedeutungserklärungen mit Angabe semantischer Relationen wie Synonymie, Antonymie und Hyponymie innerhalb der Phraseologie sowie Beziehungen zu anderen Wortschatzelementen.

- Erklärung der Bildsphäre und der Etymologie, u. U. mit Illustrationen.

- Angaben zur Frequenz und Geläufigkeit.

- Orientierung an der Sprachverwendung mit authentischen Beispielen, die die Verwendungsmöglichkeiten und die Aussprache des Phraseologismus (vgl. Hirschfeld 1996) illustrieren.

- Angaben von muttersprachlichen Entsprechungen und die Erklärung weiterer sprachenpaarbezogener Relationen.

- Die Phraseologismen sollen, zusätzlich zum semasiologischen Zugang zum Wörterbuch, onomasiologisch bzw. ideographisch nach der phraseologischen Bedeutung angeordnet werden.

- Didaktisch sinnvolle Typologien der Phraseologie.

- Interaktive Übungen zur Selbstkontrolle.

Der Erfüllung dieser Wünsche stehen mit den Möglichkeiten der Computerphraseographie keine praktischen Gründe wie Platzmangel (vgl. Ettinger 2001: 99-100) mehr im Wege.

Die Tatsache, dass die vorhandenen Wörterbücher die Bedürfnisse von Sprachlernern nicht erfüllen, ist eine Ursache für die Abwesenheit von Wörterbüchern in der Phraseodidaktik. Ein weiterer Grund könnte sein, dass die Wörterbuchbenutzungskompetenz der Sprachlerner generell zu gering ist, um Wörterbücher effektiv verwenden zu können.

Die Wörterbuchbenutzung wird (vor allem von Lexikographen) als eine selbstverständlich beherrschte Kulturtechnik betrachtet. Die Angemessenheit dieser Annahme mag für elementare Nachschlagsituationen zutreffen. Beobachtungen im universitären und schulischen Be- 
reich zeigen jedoch, daß der angemessene und qualifizierte Umgang mit Wörterbüchern, die sogenannte Wörterbuchkultur, im deutschsprachigen Raum Defizite aufweist. (Schlaefer 2002: 124)

Eine erfolgreiche Wörterbuchdidaktik vermittelt den Wörterbuchbenutzern Kompetenzen, die sie in die Lage versetzen, Wörterbücher nicht nur erfolgreich zu benutzen, sondern auch in Relation zu den eigenen Bedürfnissen und Benutzungszwecken zu evaluieren. Diese Fähigkeiten werden mit der zunehmenden Komplexität von Wörterbüchern, u. a. durch den Einsatz von neuen Medien, immer wichtiger. Der Wörterbuchbenutzer muss zunehmend selbst wissen, wie er durch verschiedene Zugangs- und Suchmöglichkeiten relevante Informationen in einem Wörterbuch findet. Für die Selbstständigkeit, die so gefordert wird, braucht der Wörterbuchbenutzer verlässliche Kriterien zur Evaluierung der Qualität von Wörterbüchern in Relation zur Wörterbuchbenutzungssituation. (Es gibt eine Fülle von beobachtenden Arbeiten zur Benutzung von Wörterbüchern, hier wird stellvertretend auf die umfangreiche Arbeit von Wiegand (1998) und das Lehrbuch von Engelberg/Lemnitzer (2001) hingewiesen).

Die Wörterbuchdidaktik ist ein bisher wenig beachtetes Forschungsgebiet in der Phraseodidaktik. Dies gilt auch umgekehrt; die Phraseodidaktik und die Phraseographie sind wenig beachtete Themen in der Wörterbuchdidaktik. Es ist anzunehmen, dass die Abwesenheit der Phraseographie in der Phraseodidaktik zumindest teilweise im Zusammenhang mit generellen Defiziten in der Wörterbuchdidaktik steht.

\section{Perspektiven}

Wenn man davon ausgeht, dass die Zukunft in der Lexikographie ein umfassendes, polyfunktionales lexikalisches Informationssystem mit allen Informationen zu einer Sprache bringen wird, das kontrastiv aufgearbeitete multilinguale Schnittstellen zu lexikalischen Informationssystemen in anderen Sprachen beinhaltet, dann ist die Phraseologie als ein Teilsystemjeder Sprache in ein solches lexikalisches Informationssystem $\mathrm{zu}$ integrieren. Eine Voraussetzung für eine gelungene und benutzeradäquate Integration ist aber eine solide Lexikographie- und Phraseologieforschung. Die Ergebnisse aus dieser Forschung, einerseits in Form von Wörterbuch- oder Datenbankkonzepten und andererseits als konkrete Analysen und Beschreibungen der Sprache, bilden die Grundlage für 
phraseographische Arbeiten. Dabei soll die Wichtigkeit "niedriger Empirie" (Dobrovol'skij 2002: 429), der Beschreibung und Analyse von Phraseologismen, ausdrücklich betont werden, denn ein Wörterbuch kann nur so gut werden, wie die Qualität des verwendeten sprachlichen Datenbestandes.

\section{Literatur und Internetseiten}

\subsection{Literatur}

Andersen, Stig Toftgaard 2001: Talemåder i dansk. Ordbog over idiomer. Kopenhagen: Gyldendal.

Babillon, Laurence 2001: Übungstypologie zur Phraseologie des Französischen. In M. Lorenz-Bourjot und H.-H. Lüger (Hg.): Phraseologie und Phraseodidaktik. Wien: Edition Praesens, 105-136.

Baur, Rupprecht, S./Chlosta, Christoph 1996: Das Projekt "Sprichwörter-Minima im Deutschen und Kroatischen": What is worth doing - do it well! In Muttersprache 2, 162-179.

Bergenholtz, Henning/Tarp, Sven 2003: Two opposing theories: On H.E. Wiegand's recent discovery of lexicographic functions. In Hermes, Journal of Linguistics 31, 171-196.

Bergenholtz, Henning 1997: Polyfunktionale ordbøger. In LexicoNordica 4, 15-28.

Braasch, Anna 1988: Zur lexikographischen Kodifizierung von Phrasemen in einsprachigen deutschen Wörterbüchern aus der Sicht eines ausländischen Wörterbuchbenutzers. In K. Hyldgaard-Jensen und A. Zettersten (Hg.): Symposium on Lexicography IV. Proceedings of the Fourth International Symposium on Lexicography April 20-22, 1988 at the University of Copenhagen. Tübingen: Max Niemeyer Verlag, 83100.

Burger, Harald 1998: Phraseologie: eine Einführung am Beispiel des Deutschen. Berlin: Erich Schmidt Verlag.

Čermák, František 2003: Paremiological Minimum of Czech: The Corpus Evidence. In H. Burger, A. Häcki Buhofer und G. Gréciano (Hg.): Flut von Texten - Vielfalt der Kulturen. Ascona 2001 zur Methodologie und Kulturspezifik der Phraseologie. Baltmannsweiler: Schneider Verlag Hohengehren, 15-31.

Cheon, Mi-Ae 1998: Zur Konzeption eines phraseologischen Wörterbuchs für den Fremdsprachler. Tübingen: Max Niemeyer Verlag.

Colson, Jean-Pierre 2003: Corpus Linguistics and Phraseological Statistics: a few Hypotheses and Examples. In H. Burger, A. Häcki Buhofer und G. Gréciano (Hg.): Flut von Texten - Vielfalt der Kulturen. Ascona 2001 zur Methodologie und Kulturspezifik der Phraseologie. Baltmannsweiler: Schneider Verlag Hohengehren, $47-$ 59. 
Cowie, Anthony P. 2003: Exploring native-speaker knowledge of phraseology: informant testing or corpus research? In H. Burger, A. Häcki Buhofer und G. Gréciano (Hg.): Flut von Texten - Vielfalt der Kulturen. Ascona 2001 zur Methodologie und Kulturspezifik der Phraseologie. Baltmannsweiler: Schneider Verlag Hohengehren, 73-81.

Dobrovol'skij, Dmitrij 1997a: Idiome im mentalen Lexikon: Ziele und Methoden der kognitivbasierten Phraseologieforschung. Trier: WVT Wissenschaftlicher Verlag.

Dobrovol'skij, Dmitrij 1997b: Kontrastive Idiomatik Deutsch-Russisch: zur lexikographischen Konzeption. In G. Gréciano (Hg.): Phraseme in Kontext und Kontrast. Bochum: Universitätsverlag Dr. N. Brockmeyer, 45-59.

Dobrovol'skij, Dmitrij 1999: Kulturelle Spezifik in der Phraseologie: allgemeine Probleme und kontrastive Aspekte. In A. Sabban (Hg.): Phraseologie und Übersetzen. Phraseologie und Übersetzen: Phrasemata II. Bielefeld: Aisthesis, 41-58.

Dobrovol'skij, Dmitrij 2002: Phraseologie als Datenbank. In D. Hartmann und J. Wirrer (Hg.): Wer A sägt, muss auch B sägen. Beiträge zur Phraseologie und Sprichwortforschung aus dem Westfälischen Arbeitskreis. Baltmannsweiler: Schneider Verlag Hohengehren, 429-432.

Drosdowski, Günther/Scholze-Stubenrecht, Werner 1998: DUDEN 11. Redewendungen und sprichwörtliche Redensarten. Wörterbuch der deutschen Idomatik. Mannheim: Dudenverlag.

Ďurčo, Peter 2001: Bekanntheit, Häufigkeit und lexikographische Erfassung von Sprichwörtern. Zu parömiologischen Minima für DaF. In A. Häcki Buhofer, H. Burger und L. Gautier (Hg.): Phraseologiae Amor. Aspekte europäischer Phraseologie. Baltmannsweiler: Schneider Verlag Hohengehren, 99-106.

Eismann, Wolfgang 1995: Pragmatik und kulturelle Spezifik als Problem der Äquivalenz von Phraseologismen. In R. S. Baur und Ch. Chlosta (Hg.): Von der Einwortmetapher zur Satzmetapher. Bochum: Universitätsverlag Dr. N. Brockmeyer, 95119.

Engelberg, Stefan/Lemnitzer, Lothar 2001: Lexikographie und Wörterbuchbenutzung. Tübingen.

Ettinger, Stefan 1998: Einige Überlegungen zur Phraseodidaktik. In W. Eismann (Hg.): EUROPHRAS 95. Bochum: Universitätsverlag Dr. N. Brockmeyer, 201-217.

Ettinger, Stefan 2001: Vom Lehrbuch zum autonomen Lernen. Skizze eines phraseologischen Grundkurses für Französisch. In M. Lorenz-Bourjot und H.-H. Lüger (Hg.): Phraseologie und Phraseodidaktik. Wien: Edition Praesens, 87-104.

Farø, Ken 2004a: Idiomer på nettet: Den danske idiomordbog og fraseografien (http:// www.idiomordbogen.dk). In Hermes, Journal of Linguistics 32, 201-235.

Farø, Ken 2004b: Am Anfang. Zur Erforschung der dänisch-deutschen Idiomatik. In J. Wirrer und Cs. Földes (Hg.): EUROPHRAS 2002. Phraseologismen als Gegenstand sprach- und kulturwissenschaftlicher Forschung. Baltmannsweiler: Schneider Verlag Hohengehren, 105-117. 
Farø, Ken 2005: Danish Phraseography. In H. Burger, D. Dobrovol'skij, N. Norrick und P. Kühn (Hg.): Phraseology/Phraseologie. An International Handbook. (Serie HSK). Berlin, New York: Walter de Gruyter (im Druck).

Farø, Ken in Vorb., a: Culture-Specific Idioms? In Jansen, Klitgaard und Pedersen (Hg.): Proceedings of the International Symposium on Translation in Copenhagen 2004. (= Perspectives).

Farø, Ken in Vorb.b: Dogmatismus, Skeptizismus, Nihilismus und Pragmatismus: 4 Haltungen zur Übersetzung von Idiomen. Erscheint In Tagungsband EUROPHRAS in Basel 2004.

Feilke, Helmuth 1993: Sprachlicher Common sense und Kommunikation. In Der Deutschunterricht 6, 6-21.

Fellbaum, Christiane/Kramer, Undine/Neumann, Gerald in Vorb.: Korpusbasierte lexikografische Erfassung und linguistische Analyse deutscher Idiome. Erscheint In Tagungsband EUROPHRAS in Basel 2004.

Filipenko, Tat'jana V. 2002: Beschreibung der Idiome in einem zweisprachigen Idiomatik-Wörterbuch (Deutsch-Russisch). In "Das Wort”, Germanistisches Jahrbuch der GUS. 43-62.

Fleischer, Wolfgang 1992: Zur lexikographischen Beschreibung deutscher Phraseologismen mit pronominaler Komponente. In J. Korhonen (Hg.): Untersuchungen zur Phraseologie des Deutschen und anderer Sprachen: einzelsprachlich - kontrastiv - vergleichend. Internationale Tagung in Turku vom 06. bis 07. September 1991. Frankfurt am Main u. a.: Peter Lang, 23-38.

Földes, Csaba 1996: Gesucht und gefunden? Idiomlexika und Deutsch als Fremdsprache. In Fremdsprache Deutsch 15, 64-67.

Fraas, Claudia/Haß-Zumkehr, Ulrike 1998: Vom Wörterbuch zum lexikalischen Informationssystem. LEKSIS - ein neues Projekt des Instituts für deutsche Sprache. In Deutsche Sprache 26/4, S. 289-303.

Friðjónsson, Jón G. 1993: Mergur málsins. Íslensk orðatiltceki. Uppruni, saga og notkun. Reykjavík: Örn og Örlygur.

Giora, Rachel 1997: Understanding figurative and literal language: The graded salience hypothesis. In Cognitive Linguistics 8 (3), 183-206.

Glaap, Albert-Reiner 1979: Idioms im Englischunterricht - kontextualisiert, sachfeldbezogen, kontrastiv. In Die Neueren Sprachen 78:6, 485-498.

Gréciano, Gertrud/Rothkegel, Annely 1995: CONPHRAS - Phraséologie Contrastive / Kontrastive Phraseologie im Rahmen von PROCOPE (Programme de Coopération Scientifique). In R. S. Baur und Ch. Chlosta (Hg.): Von der Einwortmetapher zur Satzmetapher. Bochum: Universitätsverlag Dr. N. Brockmeyer, 191-202.

Gréciano, Gertrud 1997: Zum Geleit. Zum Projekt CONPHRAS. In G. Gréciano (Hg.): Phraseme in Kontext und Kontrast. Bochum: Universitätsverlag Dr. N. Brockmeyer, $1-7$. 
Hallsteinsdóttir, Erla 2001: Das Verstehen idiomatischer Phraseologismen in der Fremdsprache Deutsch. Hamburg: Verlag Dr. Kovač. [http://www.verlagdrkovac.de/ 0435_volltext.htm]

Hallsteinsdóttir, Erla 2003: Das Verstehen idiomatischer Phraseologismen in der Fremdsprache Deutsch. In H. Burger, A. Häcki Buhofer und G. Gréciano (Hg.): Flut von Texten - Vielfalt der Kulturen. Ascona 2001 zur Methodologie und Kulturspezifik der Phraseologie. Baltmannsweiler: Schneider Verlag Hohengehren, 357-367.

Hallsteinsdóttir, Erla 2004: En kort oversigt over islandsk $\leftrightarrow$ tysk leksikografi. In LexicoNordica 11, 51-65.

Hallsteinsdóttir, Erla im Druck: Vom Wörterbuch zum Text zum Lexikon. In U. Fix, G. Lerchner, M. Schröder und H. Wellmann (Hg.): Zwischen Lexikon und Text - lexikalische, stilistische und textlinguistische Aspekte.

Hallsteinsdóttir, Erla in Vorb., a: A bilingual electronic dictionary of idioms. Erscheint In Selected Papers from the 12th International Symposium on Lexicography (Terminology and Lexicography Research and Practice).

Hallsteinsdóttir, Erla in Vorb., b: Konzeption und Erstellung einer computergestützten zweisprachigen Phraseologiesammlung Isländisch - Deutsch. Erscheint In Tagungsband EUROPHRAS in Basel 2004.

Harras, Gisela 1997: Idiome. In Klaus Peter Konerding und Andrea Lehr (Hg.): Linguistische Theorie und lexikographische Praxis. Symposiumsvorträge, Heidelberg 1996. Tübingen: Max Niemeyer Verlag, 51-60.

Hartmann, Dietrich 2002: Ein Plädoyer für lexikologische Struktur und Lexikographie phraseologischer Wortschätze. In D. Hartmann und J. Wirrer (Hg.): Wer A sägt, muss auch B sägen. Beiträge zur Phraseologie und Sprichwortforschung aus dem Westfälischen Arbeitskreis. Baltmannsweiler: Schneider Verlag Hohengehren, 433437.

Häusermann, Jürg 1977: Phraseologie. Hauptprobleme der deutschen Phraseologie auf der Basis sowjetischer Forschungsergebnisse. Tübingen: Max Niemeyer Verlag.

Herzog, Annelies/Michel, Arthur/Riedel, Herbert 1976: Deutsche idiomatische Wendungen für Ausländer. Eine Auswahl mit Beispielen. Leipzig: Verlag Enzyklopädie.

Hessky, Regina 1987: Phraseologie: Linguistische Grundfragen und kontrastives Modell Deutsch-Ungarisch. Tübingen: Max Niemeyer Verlag.

Hessky, Regina 1992: Aspekte der Verwendung von Phraseologismen im Unterricht Deutsch als Fremdsprache. In Fremdsprachen Lehren und Lernen (Jg. 21), 159168.

Hessky, Regina 1997a: Einige Fragen der Vermittlung von Phraseologie im Unterricht Deutsch als Fremdsprache. In R. Wimmer und F.-J. Berens (Hg.): Wortbildung und Phraseologie. Tübingen: Gunter Narr Verlag.

Hessky, Regina 1997b: Feste Wendungen - ein heißes Eisen? Einige phraseodidaktische Überlegungen für den DaF-Unterricht. In Deutsch als Fremdsprache 3, 139-143. 
Hessky, Regina 1999: Phraseologie: Vermittlungsinstanz zwischen Sprachsystem und Sprachverwendung. In N.F. Bravo, I. Behr und C. Rozier (Hg.): Phraseme und typisierte Rede. Tübingen: Stauffenburg, 233-241.

Hessky, Regina/Ettinger, Stefan 1997: Deutsche Redewendungen. Ein Wörter- und Übungsbuch für Fortgeschrittene. Tübingen: Gunter Narr Verlag.

Hirschfeld, Ursula 1996: Der Ton macht die Musik. Phonetik und Phraseologie. In Fremdsprache Deutsch 15, 31-35.

Holzinger, Herbert J. 1998: Kontrastive Phraseologie und Deutsch als Fremdsprache für Spanischsprechende. In Eismann, W. (Hg.): EUROPHRAS 95. Bochum: Universitätsverlag Dr. N. Brockmeyer, 337-350.

Jensen, Viggo 1982: 1000 aktuelle talemåder på dansk, engelsk og tysk. Kopenhagen: Chr. Erichsen.

Jesensek, Vida in Vorb.: Zum Problem der Äquivalenz in mehrsprachigen phraseologischen Wörterbüchern. Erscheint In Tagungsband EUROPHRAS in Basel 2004

Jónsson, Jón H. 1998: Glíman við orðasamböndin. In Orð og tunga 4, 17-24.

Jónsson, Jón H. 2001a: Staða orðasambanda í orðabókarlýsingu. In Orð og tunga 5, 61-86.

Jónsson, Jón H. 2001b: Orðastaður. Orðabók um íslenska málnotkun. Reykjavík: JPV Útgáfa.

Jónsson, Jón H. 2002: Orðaheimur. Íslensk hugtakaorðabók með orða- og orðasambandaskrá. Reykjavík: JPV Útgáfa.

Keil, Martina 1997: Wort für Wort. Repräsentation und Verarbeitung verbaler Phraseologismen (Phraseo-Lex). Tübingen: Max Niemeyer Verlag.

Kempcke, Günter 1987: Theoretische und praktische Probleme der Phraseologiedarstellung in einem synchronischen einsprachigen Bedeutungswörterbuch. In J. Korhonen (Hg.): Beiträge zur allgemeinen und germanistischen Phraseologieforschung. Internationales Symposium in Oulu 13. - 15. Juni 1986. Oulu: Universität Oulu, 155-164.

Kim-Werner, Samhwa 1998: Phraseologismen unter zweisprachigem lexikographischen Aspekt. In D. Hartmann (Hg.): "Das geht auf keine Kuhaut" - Arbeitsfelder der Phraseologie. Bochum: Universitätsverlag Dr. N. Brockmeyer, 149-167.

Kjær, Anne Lise 1987: Zur Darbietung von Phraseologismen in einsprachigen Wörterbüchern des Deutschen aus der Sicht ausländischer Textproduzenten. In J. Korhonen (Hg.): Beiträge zur allgemeinen und germanistischen Phraseologieforschung. Internationales Symposium in Oulu 13. - 15. Juni 1986. Oulu, 165-181.

Koller, Werner 1987: Überlegungen zu einem Phraseologie-Wörterbuch für Fremdsprachenunterricht und Übersetzungspraxis. In H. Burger und R. Zett (Hg.): Aktuelle Probleme der Phraseologie. Frankfurt am Main u. a.: Peter Lang, 109-120.

Koller, Werner 1994: Phraseologismen als Übersetzungsproblem. In B. Sandig (Hg.): EUROPHRAS 92. Bochum: Universitätsverlag Dr. N. Brockmeyer, 351-373. 
Korhonen, Jarmo 2003: Deutsch-finnische Phraseologie in neuerer lexikografischer Anwendung. In H. Burger, A. Häcki Buhofer und G. Gréciano (Hg.): Flut von Texten Vielfalt der Kulturen. Ascona 2001 zur Methodologie und Kulturspezifik der Phraseologie. Baltmannsweiler: Schneider Verlag Hohengehren, 490-501.

Korhonen, Jarmo 1991: Konvergenz und Divergenz in deutscher und finnischer Phraseologie. Zugleich ein Beitrag zur Erläuterung der Verbreitung und Entlehnung von Idiomen. In Chr. Palm (Hg.) EUROPHRAS 90. Akten der internationalen Tagung zur germanistischen Phraseologieforschung Aske/Schweden vom 12. bis 15. Juni 1990. Uppsala: Almqvist \& Wiksell, 123-137.

Korhonen, Jarmo 1987: Überlegungen zum Forschungsprojekt "Kontrastive Verbidiomatik Deutsch-Finnisch. In J. Korhonen (Hg.): Beiträge zur allgemeinen und germanistischen Phraseologieforschung. Internationales Symposium in Oulu 13. - 15. Juni 1986. Oulu: Universität Oulu, 1-22.

Korpi-Käkelä, Jaana (unter Mitarbeit von Irma Hyvärinen) 1996: Der Appetit kommt beim Essen. Ein Unterrichtspaket aus Finnland. In Fremdsprache Deutsch 15, 4751.

Kromann, Hans-Peder 1987: Zur Typologie und Darbietung der Phraseologismen in Übersetzungswörterbüchern. In J. Korhonen (Hg.): Beiträge zur allgemeinen und germanistischen Phraseologieforschung. Internationales Symposium in Oulu 13. 15. Juni 1986. Oulu: Universität Oulu, 183-192.

Kühn, Peter 1985: Phraseologismen und ihr semantischer Mehrwert. In Sprache und Literatur in Wissenschaft und Unterricht 56, 37-46.

Kühn, Peter 1989: Phraseologie und Lexikographie: zur semantischen Kommentierung phraseologischer Einheiten im Wörterbuch. In H. E. Wiegand (Hg.): Wörterbücher in der Diskussion. Vorträge aus dem Heidelberger Lexikographischen Kolloquium. Tübingen: Max Niemeyer Verlag, 133-151.

Kühn, Peter 1992: Phraseodidaktik. Entwicklungen, Probleme und Überlegungen für den Muttersprachenunterricht und den Unterricht Deutsch als Fremdsprache. In Fremdsprachen lehren und lernen 21, 169-186.

Kühn, Peter 1993: Aus dem Bilderbuch der deutschen Sprache. Die verkürzte Betrachtung der Phraseologismen in Sprachbüchern - Bestandsaufnahme und Perspektiven. In Der Deutschunterricht 6, 58-77.

Kühn, Peter 1994: Pragmatische Phraseologie: Konsequenzen für die Phraseographie und Phraseodidaktik. In. B. Sandig (Hg.): EUROPHRAS 92: Tendenzen der Phraseologieforschung. Bochum: Universitätsverlag Dr. N. Brockmeyer, 411-428.

Matešić, Josip 1983: Zum Terminus und zur Definition der 'phraseologischen Einheit'. In J. Matešić (Hg.): Phraseologie und ihre Aufgaben. Heidelberg: Groos.

Moon, Rosamund 1999: Needles and haystacks, idioms and corpora: Gaining insights into idioms, using corpus analysis. In Th. Herbst und K. Popp (Hg.): The Perfect Learners'Dictionary. Tübingen: Max Niemeyer Verlag, 265-281. 
Mudersbach, Klaus 1998: Ein Vorschlag zur Beschreibung von Phrasemen auf der Basis eines universalen pragmatischen Modells. In H. E. Wiegand (Hg.): Wörterbücher in der Diskussion III. Vorträge aus dem Heidelberger Lexikographischen Kolloquium. Tübingen: Max Niemeyer Verlag, 319-347.

Ófeigsson, Jón 1982: Dýzk-íslenzk orðabók. Deutsch-Isländisches Wörterbuch. Reykjavík: Almenna Bókafélagið (3. Aufl.).

Pamies, Antonio/Balmacz, Martina/Iňesta, Eva 1998: AUTOFRAS: an onomasiological tool for multi-lingual phraseology. In P. Ďurčo (Hg.): EUROPHRAS 97: Phraseology and Paremiology. Bratislava: Akadémia PZ, 267-275.

Piirainen, Elisabeth 1999: Falsche Freunde in der Phraseologie des Sprachenpaares Deutsch - Niederländisch. In A. Sabban (Hg.): Phraseologie und Übersetzen: Phrasemata II. Bielefeld: Aisthesis, 187-204.

Piirainen, Elisabeth 2000a: Phraseologie der westmünsterländischen Mundart. Teil 1. Semantische, kulturelle und pragmatische Aspekte dialektaler Phraseologismen. Baltmannsweiler: Schneider Verlag Hohengehren.

Piirainen, Elisabeth 2000b: Phraseologie der westmünsterländischen Mundart. Teil 2. Lexikon der westmünsterländischen Redensarten. Baltmannsweiler: Schneider Verlag Hohengehren.

Pilz, Klaus Dieter 1995: Duden 11. Redewendungen (...) - Das anhaltende Elend mit den phraseologischen Wörterbüchern (Phraseolexika). In R. S. Baur und Chr. Chlosta (Hg.): Von der Einwortmetapher zur Satzmetapher. Akten des Westfälischen Arbeitskreises Phraseologie/Parömiologie. Bochum: Universitätsverlag Dr. N. Brockmeyer, 305-320.

Pilz, Klaus Dieter 2002: Vorschläge für ein Phraseolexikon der deutschen Sprache. In D. Hartmann und J. Wirrer (Hg.): Wer A sägt, muss auch B sägen. Beiträge zur Phraseologie und Sprichwortforschung aus dem Westfälischen Arbeitskreis. Baltmannsweiler: Schneider Verlag Hohengehren, 299-311.

Pilz, Klaus Dieter 1987: Allgemeine und phraseologische Wörterbücher. Brauchen wir überhaupt phraseologische Wörterbücher? In Korhonen, J. (Hg.): Beiträge zur allgemeinen und germanistischen Phraseologieforschung. Internationales Symposium in Oulu 13. - 15. Juni 1986. Oulu: Universität Oulu, 129-154.

Sajankova, Monika in Vorb.: Phraseologie im Unterricht. In Tagungsband EUROPHRAS in Basel 2004.

Schlaefer, Michael 2002: Lexikologie und Lexikographie. Eine Einführung am Beispiel deutscher Wörterbücher. BerlIn Erich Schmidt Verlag.

Schreiter, Ina 1996: Grünes Licht für Redewendungen. Bericht über ein Projekt. In Fremdsprache Deutsch 15, 54-56.

Stantcheva, Diana 1999: Zum Stellenwert der Phraseologie im einsprachigen deutschen Bedeutungswörterbuch des 20. Jahrhunderts. In Linguistik online 3, 2/99. [www.linguistik-online.de] 
Tarp, Sven 1998: Leksikografien på egne ben. Fordelingsstrukturer og byggedele i et brugerorienteret perspektiv. In Hermes, Journal of Linguistics 21, 121-137.

Tarp, Sven 2004: Hvad er en bilingval ordbog? In LexicoNordica 11, 5-22.

Walker, Herbert A. 2003: Ein Vorschlag für die Darbietung von Phraseologismen in zweisprachigen Verblexika am Beispiel eines deutsch-portugiesischen Verbwörterbuchs. In H. Burger, A. Häcki Buhofer und G. Gréciano (Hg.): Flut von Texten Vielfalt der Kulturen. Ascona 2001 zur Methodologie und Kulturspezifik der Phraseologie. Baltmannsweiler: Schneider Verlag Hohengehren, 503-512.

Wiegand, Herbert Ernst 1998: Wörterbuchforschung. Untersuchungen zur Wörterbuchbenutzung, zur Theorie, Geschichte, Kritik und Automatisierung der Lexikographie. 1. Teilband. Berlin, New York: Walter de Gruyter.

Wotjak, Barbara 1992a: Verbale Phraseologismen in System und Text. Tübingen: Max Niemeyer Verlag.

Wotjak, Barbara 1992b: Probleme einer konfrontativen Phraseologieforschung am Beispiel verbaler Phraseolexeme (PL). In Korhonen, J. (Hg.): Untersuchungen des Deutschen und anderer Sprachen: einzelsprachspezifisch - kontrastiv - vergleichend. Internationale Tagung in Turku vom 06. bis 07. September 1991. Frankfurt am Main u. a.: Peter Lang, 39-60.

Wotjak, Barbara 1996: Redewendungen und Sprichwörter. Ein Buch mit sieben Siegeln? Einführung in den Themenschwerpunkt. In Fremdsprache Deutsch 15, 4-9.

Wotjak, Barbara 2001: Phraseologismen im neuen Lernerwörterbuch - Aspekte der Phraseologiedarstellung im de Gruyter-Wörterbuch Deutsch als Fremdsprache. In A. Häcki Buhofer und H. Burger (Hg.): Phraseologiae Amor. Aspekte europäischer Phraseologie. Baltmannsweiler: Schneider Verlag Hohengehren, 263-279.

Zöfgen, Ekkehard 1994: Lernerwörterbücher in Theorie und Praxis. Ein Beitrag zur Metalexikographie mit besonderer Berücksichtigung des Französischen. Tübingen: Max Niemeyer Verlag.

\subsection{Internetseiten}

Die hier aufgeführten Internetseiten stellen, zusätzlich zu den im Artikel schon erwähnten Internetangeboten, eine Auswahl von den Seiten dar, die Bibliographien und andere Materialien zu Phraseographie, Phraseologie und Parömiologie anbieten.

Das von der Alexander von Humboldt Stiftung geförderte Projekt "Kollokationen im Wörterbuch" (Leitung: Christiane Fellbaum) hat eine sehr ausführliche Online-Bibliographie zur Phraseologie:

www.bbaw.de/forschung/kollokationen/bibliography/a.html

EUROPHRAS bietet eine Bibliographie zur Phraseologie mit neueren Publikationen der EUROPHRAS-Mitglieder: www.europhras.unizh.ch/

EURALEX hat eine ausführliche Bibliographie zur Phraseologie (letzte Aktualisierung 2003): www.ims.uni-stuttgart.de/euralex/bibweb/index.html 
Wolfgang Mieders Bibliographien zu Phraseologie und Parömiologie www.deproverbio.com/DPjournal/home.html

Auf Peter Ďurčos Homepage findet man eine Bibliographie zur slawischen Phraseologieforschung: www.ucm.sk/FF/Slovensky/Katedry/nemcina/final_dt/public/durco.htm

Teodor Flontas Internetportal zu Phraseologie bietet weiterführende Links zu Phraseologie im Internet: www.utas.edu.au/docs/flonta/PHRASEOLOGY/

Peter Kühns Homepage bietet u. a. eine Demoversion eines Hypertextschulwörterbuchs mit Phraseologismen: www.daf.uni-trier.de/mitarbeiter/material/swb/swb.html

Manfred Sailers Homepage an der Universität in Tübingen beinhaltet eine Bibliographie zu unikalen Wörtern www.sfb441.uni-tuebingen.de/a5/bwb/index.html und eine Sammlung unikaler Wörter des Deutschen www.sfb441.uni-tuebingen.de/a5/codii/ index.xhtml

Jan-Philipp Soehns und Christine Römers Hompage an der Universität in Jena enthält eine Liste mit 190 IVPs ohne freie Lesart im Deutschen: personal.uni-jena.de/ xcr/ IVP-Web/ivp-liste-X.xml 